\title{
Making Planet Nine: Pebble Accretion at 250-750 AU in a Gravitationally Unstable Ring
}

\author{
Scott J. Kenyon \\ Smithsonian Astrophysical Observatory, 60 Garden Street, Cambridge, MA 02138 \\ e-mail: skenyon@cfa.harvard.edu \\ Benjamin C. Bromley \\ Department of Physics $\&$ Astronomy, University of Utah, 201 JFB, Salt Lake City, UT \\ 84112 \\ e-mail: bromley@physics.utah.edu
}

\begin{abstract}
We investigate the formation of icy super-Earth mass planets within a gravitationally unstable ring of solids orbiting at 250-750 AU around a $1 M_{\odot}$ star. Coagulation calculations demonstrate that a system of a few large oligarchs and a swarm of pebbles generates a super-Earth within 100-200 Myr at 250 AU and within 1-2 Gyr at 750 AU. Systems with more than ten oligarchs fail to yield super-Earths over the age of the solar system. As these systems evolve, destructive collisions produce detectable debris disks with luminosities of $10^{-5}-10^{-3}$ relative to the central star.
\end{abstract}

Subject headings: planetary systems - planets and satellites: formation - solar system: formation

\section{INTRODUCTION}

Over the past 10-15 yr, the discoveries of Sedna, $2012 \mathrm{VP}_{113}$, and other dwarf planets have renewed interest in the architecture of the outer solar system (e.g., Brown et al. 2004; Sheppard 2010; Chen et al. 2013; Trujillo \& Sheppard 2014, and references therein). Today, several fairly large $(R \approx 200-1000 \mathrm{~km})$ dwarf planets are known to have orbits with semimajor axis $a \gtrsim 150 \mathrm{AU}$, eccentricity $e \gtrsim 0.7$, perihelion distance $q_{p} \geq 30 \mathrm{AU}$, argument of perihelion $\omega \approx 310^{\circ}$, and longitude of perihelion $\varpi \approx 71^{\circ}$. The observed distributions of $\omega$ and $\varpi$ for these objects are statistically unlikely (e.g., Truijllo \& Sheppard 2014; de la Fuente Marcos \& de la Fuente Marcos 2014; Batygin \& Brown 2016). 
Although dynamical interactions between the Sun and a passing star can produce objects on highly eccentric orbits like Sedna and $2012 \mathrm{VP}_{113}$ (e.g., Morbidelli \& Levison 2004a; Kenyon \& Bromley 2004c; Jílková et al. 2015; Li \& Adams 2016), torques from Jupiter and the other gas giants randomize $\omega$ and $\varpi$ on $0.1-1$ Gyr time scales (Gomes et al. 2006; Lykawka \& Mukai 2008; Trujillo \& Sheppard 2014; Batygin \& Brown 2016). A super-Earth mass planet at $a \approx 200-1000$ AU can maintain the observed distributions of $\omega$ and $\varpi$ for the age of the solar system (Truillo \& Sheppard 2014; de la Fuente Marcos \& de la Fuente Marcos 2014; Iorio 2014; Batygin \& Brown 2016; Brown \& Batygin 2016). This planet might also generate a set of trans-Neptunian objects with high orbital inclination (e.g., Gladman et al. 2009; Rabinowitz et al. 2013), account for the properties of some comets (e.g., Matese et al. 1999; Gomes et al. 2006; Lykawka \& Mukai 2008), and perhaps improve the residuals in model fits to the orbit of Saturn (e.g., Fienga et al. 2016, and references therein).

To explore the origin of a possible 'planet nine', we have previously considered two broad options (Bromley \& Kenyon 2014; Kenyon \& Bromley 2015). In one mechanism, the protoplanets destined to become Jupiter, Saturn, Uranus, and Neptune scatter a lower mass, icy protoplanet from 5-15 AU to several hundred AU. Interactions with the gaseous disk then circularize the orbit. For plausible initial conditions, super-Earth mass planets achieve orbits similar to that proposed for planet nine. In another picture, the gaseous disk leaves behind a ring of solid material at 100-250 AU. Collisional evolution within the ring produces superEarth mass planets on 1-2 Gyr time scales. In principle, the orbital parameters of planet nine can distinguish between these two options.

Aside from applications to the outer solar system, these results have broad implications for the architectures of exoplanetary systems. The process of scattering, orbit circularization, and subsequent growth of icy protoplanets at $a \gtrsim 20-30$ AU plausibly explains the orbits of the gas giants in HR 8799 (Marois et al.|2008), 1RXS J160929.1-210524 (Lafrenière et al. 2010), and HD 106906 b (Bailey et al. 2014). Generation of dust grains during in situ formation of a super-Earth at 100-250 AU can account for the debris disks in HD 107146 (Corder et al. 2009; Ricci et al. 2015), HD 202628 (Krist et al. 2012), and HD 207129 (Krist et al. 2010; Marshall et al. 2011).

To improve predictions for super-Earth formation at 250-750 AU, we consider an expanded set of calculations for in situ formation of planet nine. After developing the general picture for the origin of a ring of solids at $a \gtrsim 100 \mathrm{AU}$ in 92 , we outline the numerical method (\$3) and summarize the major results (\$4). We conclude with a discussion (\$5) and brief summary (\$6]). 


\section{BACKGROUND}

Opaque protoplanetary disks surround all newly-formed stars (Kenvon \& Hartmann 1995; Kenyon et al. 2008; Williams \& Cieza 2011; Andrews 2015; Tobin et al. 2015, and references therein). Most disks have radii $R_{d} \approx 10-1000 \mathrm{AU}$ and enough solid material to build a typical planetary system (see also Najita \& Kenvon 2014). On time scales of 1-10 Myr, the optically thick disk disappears (Haisch et al. 2001; Mamajek 2009; Williams \& Cieza 2011; Alexander et al. 2014); accretion from the disk onto the central star also ceases (Hartmann et al. 1998).

Current observations are consistent with several plausible paths for disk disappearance (e.g., Najita et al. 2007; Currie et al. 2009; Cieza et al. 2010; Espaillat et al. 2010; Andrews et al. 2011; Kim et al. 2013; Najita et al. 2015, and references therein). In some systems, large inner holes suggest dissipation from the inside out, as predicted by theoretical models of photoevaporating disks (e.g., Owen et al. 2012; Gorti et al. 2015). In others, material may vanish roughly simultaneously throughout the disk.

Forming super-Earth mass planets in situ at 100-1000 AU requires a large amount of solid material. Although gaseous disks often extend to 500-1000 AU, the solids in the youngest disks rarely extend beyond 50-100 AU (Birnstiel \& Andrews 2014; Tobin et al. 2015; Andrews 2015; Canovas et al. 2016, and references therein). Thus, we seek a mechanism to transport small particles radially outward. Although developing a robust calculation of radial transport is beyond the scope of this paper, we show that gas drag in a photoevaporating protoplanetary disk is a plausible mechanism for generating a ring of pebbles beyond 100 AU.

\subsection{Radial transport of small particles in protoplanetary disks}

In all protoplanetary disks, pressure causes the gas to orbit the central star somewhat more slowly than the local circular velocity (Adachi et al. 1976; Weidenschilling 1977). Solid particles then feel a headwind which drags them towards local pressure maxima (see also Youdin \& Shu 2002; Youdin \& Chiang 2004; Brauer et al. 2008; Birnstiel et al. 2010; Chiang \& Youdin 2010; Windmark et al. 2012; Garaud et al. 2013). For 1-100 cm particles, the drift velocity is roughly $0.1 \%$ of the orbital velocity. Thus, drift times are short.

When the disk has a smooth radial surface density gradient, $\Sigma \propto a^{-p}$ with $p>$ 0, small solids typically drift radially inward (e.g., Rafikov 2004; Youdin \& Chiang 2004; Chiang \& Youdin 2010, and references therein). As small particles spiral in toward the central star, they may concentrate within local pressure maxima produced by vortices or dust traps (e.g., Klahr \& Henning 1997; Chavanis 2000; Haghighipour \& Boss 2003; Heng \& Kenyon 2010; Raettig et al. 2015; Gibbons et al. 2015) or at the edges of gaps produced by massive 
planets (Ayliffe et al. 2012; Zhu et al. 2014; Gonzalez et al. 2015).

This behavior suggests a mechanism for outward drift of small particles. Consider a disk with inner edge $a_{\text {in }}$ and outer edge $a_{\text {out }}$ orbiting a central star with radius $R_{\star}$. If $a_{\text {in }} \gg R_{\star}$ and $a_{\text {out }} \gg a_{\text {in }}$, the disk surface density $\Sigma$ rises from zero at $a_{\text {in }}$ to some maximum $\Sigma_{\max }$ and then declines monotonically outward. If the disk temperature also follows a power-law, $T \propto r^{-q}$, the disk has a maximum pressure $P_{\max }$ at some $a=a_{\max }$ close to $a_{\text {in }}$. Small particles at $a_{\max } \leq a \leq a_{\text {in }}\left(a \geq a_{\max }\right)$ then drift radially outward (inward) until they reach $a_{\max }$. Now suppose $a_{i n}$ and $a_{\max }$ expand on a time scale longer than the radial drift time. If the disk maintains a pressure maximum at $a_{\max }$, particles are continuously swept from $a_{i n}$ to $a_{\max }$. Once the disk dissipates, it leaves behind a ring of small particles at the last $a_{\max }$. A sufficiently large final $a_{\max }$ enables super-Earth formation at 250-750 AU.

Photoevaporating disks offer one way to achieve this evolution. In theoretical models (e.g., Owen et al. 2012; Gorti et al. 2015, and references therein), high energy photons from the central star and the inner disk ionize material above the disk photosphere. The high temperature of this material generates a wind which removes gas from the disk. As the system evolves, the surface density of the disk declines. Eventually, mass loss creates an inner hole devoid of gas. Expansion of the inner hole gradually removes more and more material from the disk until the disk vanishes.

For plausible protostellar disks, the expansion rate of the inner edge of the disk is much smaller than the radial drift rate of small particles. Numerical calculations (Clarke et al. 2001; Gorti \& Hollenbach 2009; Owen et al. 2012, 2013; Gorti et al. 2015; Haworth et al. 2016) and observations (Calvet et al. 2005; Currie et al. 2009; Cieza et al. 2010; Andrews et al. 2011; Najita et al. 2015) indicate an expansion rate $\dot{a}_{i n} \gtrsim 10-20 \mathrm{AU} \mathrm{Myr}^{-1} \approx 5-10 \mathrm{~cm} \mathrm{~s}^{-1}$. Typical radial drift rates for $1-10 \mathrm{~cm}$ particles are $3-30 \mathrm{~m} \mathrm{~s}^{-1}$. For photoevaporation models with $\dot{a}_{i n} \lesssim 1000-2000 \mathrm{AU} \mathrm{Myr}^{-1}$, it seems plausible that the expanding inner edge of the disk can transport small particles to large radial distances.

\subsection{Dynamical Cooling and Gravitational Instability}

As the inner edge of the disk expands, we assume that turbulence within the gas prevents swept up small particles from colliding and merging into larger objects. Once the gas has dissipated, turbulence is minimal. In Kenyon \& Bromley (2015), we considered the collisional evolution of sets of mono-disperse distributions of particles covering a range of sizes, $1 \mathrm{~cm}$ to $10^{3} \mathrm{~km}$. Here, we explore outcomes when a collection of cm-sized 'pebbles' becomes gravitationally unstable and produces much larger oligarchs.

In a swarm of pebbles with velocity dispersion $c_{p}$, surface density $\Sigma$, and angular velocity $\Omega$, the system is gravitationally unstable when $c_{p} \Omega<\pi G \Sigma$ (e.g., Chiang \& Youdin 2010). Setting $\Sigma=\Sigma_{0}(a / 1 \mathrm{AU})^{-3 / 2}$, gravitationally stability requires a particle eccentricity $e \gtrsim e_{s}$, 
where

$$
e_{s} \approx 10^{-4}\left(\frac{\Sigma_{0}}{30 \mathrm{~g} \mathrm{~cm}^{-2}}\right)\left(\frac{a}{100 \mathrm{AU}}\right)^{1 / 2} .
$$

For material at 250-750 AU, the minimum $e$ for gravitational stability is $e_{s} \approx 1-3 \times 10^{-4}$.

Once the disk dissipates, pebbles with an initial $e \approx 10^{-3}$ set by disk turbulence damp very quickly. In Kenyon \& Bromley (2015), the e-folding time for collisional damping to reduce $e$ is roughly $10^{4}$ yr at $125 \mathrm{AU}$. The damping time, $t_{d} \propto P / \Sigma$, scales with $\Sigma$ and the orbital period $P$. For annuli with identical total masses in pebbles and width $\delta a=0.2 a$, the damping time is roughly $10^{5} \mathrm{yr}$ at $250 \mathrm{AU}$ and $5 \times 10^{6} \mathrm{yr}$ at $750 \mathrm{AU}$. These time scales are reasonably short, so we assume the pebbles damp into a gravitationally unstable ring.

Outcomes of gravitational instability remain uncertain (e.g., Michikoshi et al. 2007; Chiang \& Youdin 2010; Johansen et al. 2015; Simon et al. 2015, and references therein). Here, we follow Michikoshi et al. (2009, 2010) and assume that the instability produces one or more oligarchs with a size $r_{\max }$ set by the wavelength of the shortest stable mode in the swarm of pebbles. For the conditions we consider below, $r_{\max } \approx 100 \mathrm{~km}$. Once $N$ oligarchs collapse out of the swarm, they begin to accrete leftover pebbles. At the same time, pebbles begin to collide and merge into larger objects. Although this set of starting conditions is somewhat artificial, it captures the spirit of the likely evolution following gravitational instability in a ring of pebbles.

\section{PLANET FORMATION CALCULATIONS}

To investigate planet growth at 250-750 AU, we consider coagulation calculations for a single annulus with width $\delta a$ at semimajor axis a (Kenyon \& Bromley 2015, see Table 1 for a list of parameters). Solid material orbits a central star with mass $M_{\star}=1 M_{\odot}$. The annulus contains $M$ mass batches (labeled from $k=1$ to $k=M$ ) with characteristic mass $m_{k}$ and radius $r_{k}$ (Wetherill \& Stewart 1993; Kenyon \& Luu 1998). Batches are logarithmically spaced in mass, with mass ratio $\delta \equiv m_{k+1} / m_{k}$. Each mass batch contains $N_{k}$ particles with total mass $M_{k}$ and average mass $\bar{m}_{k}=M_{k} / N_{k}$. Particle numbers $N_{k}<10^{15}$ are always integers. Throughout the calculation, various algorithms use the average mass to calculate the average physical radius $\bar{r}_{k}$, collision cross-section, collision energy, and other necessary physical variables. As mass is added and removed from each batch, the number of particles, the total mass, and the average mass change (Wetherill \& Stewart 1993; Kenyon \& Luu 1998; Kenyon \& Bromley 2015, 2016).

In these calculations, we follow the evolution of particles with mass density $\rho_{p}=$ $1.5 \mathrm{~g} \mathrm{~cm}^{-3}$ and sizes ranging from a minimum $r_{\text {min }}=1 \mu \mathrm{m}$ to a maximum $r_{\max }$. The

mass spacing factor $\delta=2^{1 / 4}$. Initially, the annulus contains two mono-disperse swarms of planetesimals with initial sizes $0 ْ$ and $r_{\max }$, total surface density $\Sigma_{0}$, total mass $M_{0}$, and hor- 
izontal and vertical velocities $h_{0}$ and $v_{0}$ relative to a circular orbit. Within the swarm, the large particles contain a fraction $f_{0}$ of the initial mass $M_{0}$. The horizontal velocity depends on the orbital eccentricity, $e=1.6\left(h / v_{K}\right)^{2}$, where $v_{K}$ is the circular orbital velocity. The orbital inclination is $\sin i=\sqrt{2} v / v_{K}$.

The mass and velocity distributions of the planetesimals evolve in time due to inelastic collisions, drag forces, and gravitational encounters. This evolution depends on the solution to a coupled set of coagulation and Fokker-Planck equations which treats the outcomes of mutual collisions between all particles in all mass bins (Kenyon \& Luu 1998, 1999; Kenyon \& Bromley 2004a, 2008, 2015, 2016). For physical collisions, we adopt the particlein-a-box algorithm; the collision rate is then $n \sigma v f_{g}$, where $n$ is the number density of objects, $\sigma$ is the geometric cross-section, $v$ is the relative velocity, and $f_{g}$ is the gravitational focusing factor (Wetherill \& Stewart 1993; Kenyon \& Luu 1998). For a specific mass bin, the solutions include terms for (i) loss of mass from mergers with other objects and (ii) gain of mass from collisional debris and mergers of smaller objects.

Depending on physical conditions in the disk, we derive $f_{g}$ in the dispersion or the shear regime (Kenyon \& Luu 1998; Goldreich et al. 2004; Kenyon \& Bromley 2012; Youdin \& Kenyon 2013). To set conditions in the shear regime, we define the Hill radius

$$
R_{H}=a\left(m / 3 M_{\star}\right)^{1 / 3} .
$$

When interpreting results of the calculations, it is useful to track the Hill radius of the largest object with $m=m_{\max }$ and the relative eccentricity, $e_{r e l}=e a / R_{H}$, of small particles. When $e_{\text {rel }} \lesssim 1$ ( $\approx 1$ ), collisions with the largest object are in the shear (dispersion) regime.

For the most massive super-Earths produced in these simulations, the Hill radius is $R_{H} \approx 0.02 a$. With the annulus width $\delta a=0.2 a$, the width of the annulus is much larger than the Hill radius of the largest objects in the simulation.

Within the single annulus, the most massive protoplanets on roughly circular orbits are 'isolated' from one another (Wetherill \& Stewart 1993). Isolated protoplanets can accrete smaller objects but cannot collide with other isolated protoplanets. Our algorithm identifies the $n(n+1)$ largest objects whose combined gravitational range is smaller (larger) than $\delta a$ and establishes these $n$ objects as isolated protoplanets (Kenyon \& Luu 1998; Kenyon \& Bromley 2015).

Collision outcomes depend on the ratio $Q_{c} / Q_{D}^{\star}$, where $Q_{D}^{\star}$ is the collision energy needed to eject half the mass of a pair of colliding planetesimals to infinity and $Q_{c}$ is the center of mass collision energy (see also Wetherill \& Stewart 1993; Williams \& Wetherill 1994; Tanaka et al. 1996; Stern \& Colwell 1997; Kenvon \& Luu 1999; O’Brien \& Greenberg 2003; Kobavashi \& Tanaka 2010). A colliding pair of planetesimals with horizontal velocity $h_{1}, h_{2}$ and vertical velocity $v_{1}, v_{2}$ have relative horizontal and vertical velocities $h_{c}=\left(h_{1}^{2}+h_{2}^{2}\right)^{1 / 2}$ and $v_{c}=\left(v_{1}^{2}+v_{2}^{2}\right)^{1 / 2}$ (see also Wetherill \& Stewart 1993; Kenvon \& Luu 1998; Kenyon \& Bromley 
2004a, 2015). The escape velocity of the colliding pair is $v_{e s c}=\left(2 G m_{c} / r_{c}\right)^{1 / 2}$, where $m_{c}=m_{1}+m_{2}$ is the combined mass and $r_{c}=r_{1}+r_{2}$ is the combined radius. The center of mass collision energy is then

$$
Q_{c}=0.5 \mu\left(h_{c}^{2}+v_{c}^{2}+v_{e s c}^{2}\right) / m_{c}
$$

where $\mu=m_{1} m_{2} / m_{c}$ is the reduced mass.

Consistent with N-body simulations of collision outcomes (e.g., Benz \& Asphaug 1999; Leinhardt et al. 2008; Leinhardt \& Stewart 2009), we set

$$
Q_{D}^{\star}=Q_{b} r^{\beta_{b}}+Q_{g} \rho_{p} r^{\beta_{g}}
$$

where $Q_{b} r^{\beta_{b}}$ is the bulk component of the binding energy and $Q_{g} \rho_{p} r^{\beta_{g}}$ is the gravity component of the binding energy. For 'strong' planetesimals, we adopt $Q_{b}=2 \times 10^{5} \mathrm{erg} \mathrm{g}^{-1} \mathrm{~cm}^{0.4}$, $\beta_{b}=-0.40, Q_{g}=0.22 \mathrm{erg} \mathrm{g}^{-2} \mathrm{~cm}^{1.7}$, and $\beta_{g}=1.30$. These parameters are broadly consistent with published analytic and numerical simulations (e.g., Davis et al. 1985; Holsapple 1994; Love \& Ahrens 1996; Housen \& Holsapple 1999). At small sizes, they agree with results from laboratory (e.g., Ryan et al. 1999; Arakawa et al. 2002; Giblin et al. 2004; Burchell et al. 2005) and numerical (e.g., Leinhardt \& Stewart 2009) experiments of impacts between icy objects. For $r \approx 10-100 \mathrm{~m}$ particles with the smallest $Q_{D}^{\star}, Q_{D}^{\star}$ is a factor of $3-10$ smaller than in other studies (e.g., Bottke et al. 2010). Thus, these small planetesimals are relatively easy to break.

In some calculations, we consider ensembles of 'weak' planetesimals with $Q_{b}=10^{3} \mathrm{erg} \mathrm{g}^{-1}$ and $\beta_{b}=0$. Among these objects, small objects have negligible material strength; the binding energies of large objects are set by gravity as for strong planetesimals.

For two colliding planetesimals with masses $m_{1}$ and $m_{2}$, the mass of the merged planetesimal is

$$
m=m_{1}+m_{2}-m_{e s c},
$$

where the mass of debris ejected in a collision is

$$
m_{e s c}=0.5\left(m_{1}+m_{2}\right)\left(\frac{Q_{c}}{Q_{D}^{*}}\right)^{b_{d}}
$$

The exponent $b_{d}$ is a constant of order unity (e.g., Davis et al. 1985; Wetherill \& Stewart 1993; Kenyon \& Luu 1999; Benz \& Asphaug 1999; O’Brien \& Greenberg 2003; Leinhardt \& Stewart 2012). We adopt $b_{d}=1$ (see also Kobayashi \& Tanaka 2010; Kobayashi et al. 2010; Kenyon \& Bromley 2015).

To place the debris in the grid of mass bins, we set the mass of the largest collision fragment as

$$
m_{\max , d}=\min \left(m_{L, 0}, m_{L, 0}\left(Q_{c} / Q_{D}^{\star}\right)^{-b_{L}}\right) m_{e s c}
$$


and adopt a differential size distribution $N(r) \propto r^{-3.5}$. After placing a single object with mass $m_{\max , d}$ in the grid, we place material in successively smaller mass bins until (i) the mass is exhausted or (ii) mass is placed in the smallest mass bin. Any material left over is removed from the grid. For these calculations, we adopt $m_{L, 0}=0.2$ and $b_{L}=0$ or 1 .

As we place the debris in specific mass bins, we also redistribute the kinetic energy per unit mass of each colliding pair of planetesimals. As in Kenvon \& Luu (1998), we assume all collisions between mass batches conserve the horizontal and vertical components of kinetic energy. For an initial kinetic energy, $m_{1}\left(h_{1}^{2}+v_{1}^{2}\right)+m_{2}\left(h_{2}^{2}+v_{2}^{2}\right)$, any merged planetesimal with mass $m$ receives a fraction $m /\left(m_{1}+m_{2}\right)$ of this kinetic energy; any fragment with mass $m_{f}$ receives a fraction $m_{f} /\left(m_{1}+m_{2}\right)$. Recalling the center of mass collision energy from eq. (3), this approach assumes that the escape velocity component of the collision energy is equal to the energy required to disperse the fragments to infinity.

To compute the evolution of the velocity distribution, we also include collisional damping from inelastic collisions and gravitational interactions. For inelastic and elastic collisions, we follow the statistical, Fokker-Planck approaches of Ohtsuki (1999) and Ohtsuki et al. (2002), which treat pairwise interactions (e.g., dynamical friction and viscous stirring) between all objects. For evaluating these interactions within a single annulus, we eliminate terms to calculate the probability that objects in one annulus interact with objects in other annuli (Kenyon \& Bromley 2001, 2004b, 2008). We also compute long-range stirring from distant oligarchs (Weidenschilling 1989). At 250-750 AU, particles collide and grow on time scales much longer than the 1-5 Myr lifetime of the gaseous disk (Haisch et al. 2001; Williams \& Cieza 2011; Cloutier et al. 2014). Thus, we set the initial surface density of the gas to zero and ignore gas drag on small solids (Adachi et al. 1976; Weidenschilling 1977; Rafikov 2004).

In previous studies, we have compared results from the coagulation code with analytical models and other numerical simulations of collision rates/outcomes, collisional damping, and gravitational stirring (Kenvon \& Luu 1998; Kenvon \& Bromley 2001, 2004d, 2006; Bromley \& Kenyon 2006; Kenyon \& Bromley 2008, 2015, 2016). Our calculations yield excellent matches to these results. Typical solutions conserve mass and energy to machine accuracy. Over the $10^{6}$ timesteps in a typical 10 Gyr run, mass and energy are conserved to better than a part in $10^{10}$.

\section{EVOLUTION OF THE LARGEST OBJECTS}

To evolve a sea of planetesimals in a single annulus, we set the sizes 0 and $r_{\text {max }}$, the surface density $\Sigma_{0}$, the fraction $f_{0}$ of the initial mass in the largest objects, and the orbital elements $e_{0}$ and $i_{0}$. For these simulations, $0^{\circ}=1 \mathrm{~cm}, r_{\max }=100 \mathrm{~km}$, and $f_{0}=0.5$ to $10^{-7}$. The total mass in the annulus is $15.8 M_{\oplus}$. The surface density of an annulus with $\delta a=0.2 a$ 
is then $\Sigma_{0}=5.4 \times 10^{-3} \mathrm{~g} \mathrm{~cm}^{-2}$ at $250 \mathrm{AU}\left(6 \times 10^{-4} \mathrm{~g} \mathrm{~cm}^{-2}\right.$ at $\left.750 \mathrm{AU}\right)$. For comparison, the minimum mass solar nebula has $\Sigma \approx 30 \mathrm{~g} \mathrm{~cm}^{-2}(a / 1 \mathrm{AU})^{-3 / 2}$ (Kenyon \& Bromley 2008), which implies $\Sigma=7.6 \times 10^{-3} \mathrm{~g} \mathrm{~cm}^{-2}$ at $a=250 \mathrm{AU}\left(\Sigma=1.5 \times 10^{-3} \mathrm{~g} \mathrm{~cm}^{-2}\right.$ at $a=$ $750 \mathrm{AU}$ ). Evolution times depend inversely on the mass in solid objects (Kenyon \& Luu 1999; Kenyon \& Bromley 2002, 2008, 2010); thus, we consider only one surface density at each $a$.

In most published simulations, the initial orbital elements of planetesimals are set to match the escape velocity of the largest objects (e.g., Kobavashi et al. 2010; Weidenschilling 2010). For particles with $0 \gtrsim 10-100 \mathrm{~m}$ at $250-750 \mathrm{AU}$, the time scale to reach equilibrium is longer than the lifetime of the gaseous disk (e.g., Kenvon \& Bromley 2015). Here, we set $i_{0}=e_{0} / 2$ and adopt $e_{0}=10^{-4}$ at $250 \mathrm{AU}$ and at $750 \mathrm{AU}$. With these initial conditions, swarms of small particles are marginally gravitationally stable; accretion begins in the shear regime. For the small Hill radii of $100 \mathrm{~km}$ objects, however, initial accretion rates are fairly small. Kenyon \& Bromley (2015) demonstrate that modest changes to $e_{0}$ and $i_{0}$ have little impact on outcomes.

\subsection{Evolution at $250 \mathrm{AU}$}

In all calculations, the evolution follows a standard pattern. Large oligarchs accrete pebbles fairly slowly. The small particles also merge and grow. Along with the steady growth of an oligarch's 'feeding zone' (set by the Hill radius), dynamical friction circularizes $e$ and $i$ for the oligarchs. Gravitational focusing factors increase dramatically; runaway growth begins. During runaway growth, rapidly growing oligarchs stir up the more slowly growing pebbles. As gravitational focusing factors decline, the growth of oligarchs stalls.

When runaway growth ends, oligarchs continue to accrete small particles. Among the small particles, however, accretion and destructive collisions generate a broad size distribution with sizes ranging from $1 \mu \mathrm{m}$ to $1 \mathrm{~km}$. As the evolution proceeds, collisional damping among $0.1-1.0 \mathrm{~m}$ and smaller particles overcomes gravitational stirring by the oligarchs (see also Kenyon \& Bromley 2015, 2016). Collisions among these small particles produce larger merged objects. Destructive collisions among the merged objects allow material to cycle between large and small objects, generating a roughly steady state size distribution. In addition to cycling of mass among $1 \mu \mathrm{m}$ to $1 \mathrm{~km}$ objects, destructive collisions yield small objects $(r \lesssim 1 \mu \mathrm{m})$ which are ejected from the system. Thus, the mass of the system declines with time.

Eventually, collisional damping of small particles leads to a second phase of runaway growth for the oligarchs. During this epoch, a few oligarchs grow from 2000-4000 km to $\gtrsim 10^{4} \mathrm{~km}$. With more mass in oligarchs and less mass in small particles, gravitational stirring overcomes collisional damping. Destructive collisions among all the small particles 
then power a collisional cascade, where the small particles are ground to dust and ejected from the system. The growth of oligarchs ceases.

Fig. 1 illustrates the growth of the largest objects in a suite of calculations with strong planetesimals and various $f_{0}$. Among the oligarchs, initial accretion rates are small, $\sim$ $10^{15} \mathrm{~g} \mathrm{yr}^{-1}$. When $f_{0} \lesssim 10^{-5}$, steady growth and circularization lead to a first runaway growth phase at 4 Myr. By 10-30 Myr, oligarchs have grown by factors of 10-100. Maximum sizes are much larger in systems starting with a few oligarchs than in those starting with many oligarchs. From 50 Myr to a few Gyr, the largest oligarchs then grow slowly. When collisional damping allows a second phase of runaway growth, oligarchs reach maximum sizes of $3000 \mathrm{~km}$ to $2 \times 10^{4} \mathrm{~km}$.

In systems with a larger fraction of the mass in oligarchs, growth is much slower. When $f_{0} \gtrsim 10^{-4}$, oligarchs rapidly stir themselves and the small pebbles. Gravitational focusing factors remain small, eliminating the initial phase of runaway growth. As stirring continues, more and more material is ejected or ends up in pebbles where collisional damping can overcome stirring by the oligarchs. Once damping overcomes stirring, large gravitational focusing factors enable a strong (but very late) phase of runaway growth where oligarchs grow from $200-300 \mathrm{~km}$ to $3-10 \times 10^{3} \mathrm{~km}$ sizes. Substantial mass loss prior to runaway growth often limits the maximum sizes of oligarchs.

Despite the remarkable evolution during the second phase of runaway growth, few systems produce super-Earth mass planets on time scales shorter than the age of the solar system. Nearly all ensembles with 1-2 oligarchs yield super-Earths in 200 Myr to 1 Gyr. Once the initial number of oligarchs exceeds 10, the second runaway growth phase is either too weak or too late to enable super-Earth formation in $\lesssim 1-2$ Gyr.

When planetesimals are much weaker, growth is less dramatic in systems with $f_{0} \lesssim 10^{-5}$ (Fig. 2). As in calculations with strong planetesimals, dynamical friction between oligarchs and pebbles initiates runaway growth of oligarchs at 3-5 Myr. After another 5-15 Myr, gravitational stirring by growing oligarchs raises the $e$ and $i$ of the pebbles and dramatically reduces gravitational focusing factors. The growth of oligarchs stalls. When small planetesimals are weak and easy to break, stirring initiates the collisional cascade at an earlier epoch than in systems with strong planetesimals (see also Kenyon \& Bromley 2010). Destructive collisions then result in greater mass loss from the system. Collisional damping never overcomes gravitational stirring, preventing a second phase of runaway growth. Compared to the most massive oligarchs in systems of strong planetesimals, the most massive oligarchs in these calculations are 20-50 times less massive.

Systems with larger $f_{0}$ evolve fairly independently of the planetesimal strength. In these systems, stirring by oligarchs maintains fairly large $e$ and $i$ for pebbles and other small objects. Larger $e$ and $i$ generates more debris and more mass loss. All of these systems skip the first runaway growth phase at 3-10 Myr. As the evolution proceeds, mass loss continues. 
In most systems, though, collisional damping slowly raises gravitational focusing factors. At 1-2 Gyr, there is a short period of runaway growth which produces 1-2 objects with radii of 1000-5000 km. Continued stirring initiates a stronger collisional cascade which grinds the few remaining small objects to dust.

In all simulations of solid evolution at $250 \mathrm{AU}$, the evolution of small particles also follows a standard path (Fig. 3). Initially, all pebbles have radii of $1 \mathrm{~cm}$. After $\sim 1$ Myr, growth and debris production produce a multi-component size distribution extending from $r_{\min }=$ $1 \mu \mathrm{m}$ to roughly $1 \mathrm{~m}$. When the 'pebbles' reach sizes of $1 \mathrm{~km}$, stirring by oligarchs begins to drive a collisional cascade. Destructive collisions first eliminate the weakest planetesimals with radii of $\sim 0.1 \mathrm{~km}$. As the cascade proceeds, stirring continues to raise collision energies of large planetesimals; thus, collisions destroy progressively stronger (smaller) planetesimals. By 30 Myr, nearly all particles with radii of 1-100 m have been destroyed, producing a striking plateau in the cumulative size distribution. Debris from this population generates a power-law size distribution for pebbles with $r \lesssim 10 \mathrm{~cm}$.

Until the second phase of runaway growth begins, destructive collisions and damping maintain two prominent features of the size distribution. Among the smallest particles, the debris follows a power-law size distribution. At the large end of this distribution, there is an abrupt drop in the cumulative number of particles. The particle radius at this drop, $r_{d}$, separates regions where collisional damping $\left(r \lesssim r_{d}\right)$ and gravitational stirring $\left(r \gtrsim r_{d}\right)$ dominate the velocity evolution. At larger sizes, destructive collisions produce debris; at smaller sizes, collisions produce larger merged objects. Solid material cycles between the two regions.

Fig. 4illustrates the evolution of the 'damping front' in more detail (see also Kenvon \& Bromley 2015). At large sizes $\left(r \gtrsim r_{d}\right)$, gravitational stirring by massive oligarchs drives particle eccentricities to larger and larger values. In this example, the relative eccentricity grows from $e_{r e l} \approx 4$ to $e_{r e l} \approx 15$ as the mass of the most massive oligarch grows by a factor of 50 . Among smaller particles, collisional damping produces a sharp, factor of 10-20, drop in the relative eccentricity. As the system evolves, the damping front moves to smaller and smaller particle radii. Once these particles contain enough mass, they power a second phase of runaway growth where the most massive oligarchs reach super-Earth masses. Eventually, the mass in small particles vanishes. Gravitational stirring dominates collisional damping; the damping front disappears. All small particles then have roughly the same $e_{\text {rel }}$.

To summarize results for the complete suite of simulations, Fig. 5 shows $r_{\text {max }}$ at the end of each calculation as a function of $f_{0}, b_{L}$, and the planetesimal strength. Among calculations with weak planetesimals and $b_{L}=0$, there is a clear trend of less growth with more initial mass in large oligarchs. In these calculations, more oligarchs produce more gravitational stirring among the pebbles. With more gravitational stirring, pebbles are easier to break as they grow from $1-10 \mathrm{~cm}$ to $10 \mathrm{~m}$ to $1 \mathrm{~km}$ sizes. Collisions then produce more debris, robbing the massive oligarchs of material to grow to super-Earth sizes. 
When $b_{L}=1$ and planetesimals are weak, some calculations follow the trend established for systems with $b_{L}=0$. For simulations with $f_{0}=1-100 \times 10^{-7}$ and $f_{0} \approx 0.3-0.5$, gravitational stirring by oligarchs leads to destructive collisions which remove significant amounts of mass from the annulus. Oligarch growth then stalls at small sizes. Among the simulations with intermediate $f_{0}$, collisional damping associated with copious amounts of debris often overcomes gravitational stirring, enabling the system to retain small particles and energizing a second phase of runaway growth and the production of Earth mass planets. In these simulations, there is a broad range of outcomes: Pluto to Mars mass planets are just as likely as Earth mass planets.

To understand the long-term evolution of systems of weak planetesimals in more detail, we performed an additional set of calculations with $Q_{b}=10 \mathrm{erg} \mathrm{g}^{-1}$ and $b_{L}=1$. In these systems, planetesimals begin to break at even earlier times than those with $Q_{b}=10^{3} \mathrm{erg} \mathrm{g}^{-1}$. Mass loss is more severe; collisional damping never overcomes gravitational stirring. The variation of $m_{\max }$ with $f_{0}$ then almost precisely follows results for calculations with weak planetesimals and $b_{L}=0$ in Fig. 5, where no oligarchs reach super-Earth masses.

Within the suite of calculations with strong planetesimals, super-Earth formation is common. Nearly all simulations with $b_{L}=1$ yield a super-Earth. Although most simulations with $b_{L}=0$ produce a super-Earth, the largest objects in calculations with $f_{0}=0.3-0.5$ always remain small. Despite the overall success of these simulations, rapid super-Earth production is still rare. In all calculations with $f_{0} \gtrsim 10^{-6}$, protoplanets reach super-Earth masses on 5-10 Gyr time scales, longer than the age of the solar system. When systems begin with $\lesssim 5-10$ massive oligarchs, a single massive protoplanet can grow to super-Earth masses in 100-300 Myr.

\subsection{Evolution at $750 \mathrm{AU}$}

Within an annulus at $750 \mathrm{AU}$, oligarchs follow the same evolutionary path as at $250 \mathrm{AU}$ (Fig. 6). At the start of the calculations, $100 \mathrm{~km}$ oligarchs accrete material in the shear regime. With typical growth rates of $2-6 \times 10^{13} \mathrm{~g} \mathrm{yr}^{-1}$, it takes $100-600 \mathrm{Myr}$ for oligarchs to double in mass. As they grow, oligarchs try to stir up the much smaller pebbles. Collisional damping among the pebbles counters this stirring.

In systems with a few oligarchs $\left(f_{0} \lesssim 10^{-5}\right)$ and strong pebbles, collisional damping dominates gravitational stirring. Once oligarchs have 2-4 times their initial masses, runaway growth begins. Within a few Myr, oligarchs reach sizes of 400-4000 km. Throughout runaway growth, gravitational stirring rates also grow. Eventually, stirring overcomes damping. Runaway growth ends.

As runaway growth ends, destructive collisions among leftover pebbles and planetesimals

drive a collisional cascade. When the number of oligarchs is small $(\lesssim 10-20)$, collisional 
damping among small particles once again dominates gravitational stirring by the oligarchs. Damping powers a second phase of runaway growth, where oligarchs may reach super-Earth masses. The onset of this epoch depends on the number of oligarchs. Systems with 1-4 oligarchs reach super-Earth masses in 1-2 Gyr. Although systems with 8 or more oligarchs enter a second phase of runaway growth, this evolution never leads to super-Earth mass planets.

In systems with many oligarchs, stirring dominates damping. Oligarch masses grow only by factors of 2-3 over 1-2 Gyr, runaway growth never develops. After 10 Gyr, oligarchs reach sizes of $150-200 \mathrm{~km}$.

When pebbles at $750 \mathrm{AU}$ are weak (Fig. 7), the evolution never leads to super-Earth mass planets. Although the initial phase of runaway growth produces massive planets, the subsequent collisional cascade effectively destroys leftover pebbles and more massive planetesimals. In these systems, collisional damping is never effective enough to create the pronounced damping front observed in calculations with strong pebbles. Collisions destroy pebbles and smaller objects faster than oligarchs can accrete them. Thus, growth stalls at much smaller masses.

For calculations with strong or weak planetesimals, the timing of the first phase of runaway growth always occurs 30-40 times later at 750 AU (120-150 Myr) than at 250 AU (4 Myr). When $\stackrel{0}{0}, r_{\max }, f_{0}, e_{0}$, and $i_{0}$ are identical, the time for runaway growth to produce objects with a fixed size depends on the orbital period, surface density, and the gravitational focusing factor $t \propto P / f_{g} \Sigma$ (e.g., Lissauer 1987; Kenyon \& Bromley 2008). Given our starting conditions, initial gravitational focusing factors at $250 \mathrm{AU}$ and at $750 \mathrm{AU}$ are roughly equal; $P / \Sigma$ is roughly 45 times larger at $750 \mathrm{AU}$ than at $250 \mathrm{AU}$. Despite stochastic variations in the collision, damping, and stirring rates, the predicted factor of 45 difference in the timing of runaway growth is remarkably close to the factor of 30-40 difference of our calculations.

\subsection{Luminosity Evolution}

Aside from detecting planets by direct imaging (e.g., Marois et al. 2008; Lafrenière et al. 2010; Lagrange et al. 2010; Bailey et al. 2014; Currie et al. 2014a.b), scattered light and thermal emission from small particles are the only diagnostic of the long-term evolution of protoplanets at large $a$. In our calculations, we track the size distribution of $1 \mu \mathrm{m}$ and larger particles. Relative to the luminosity of the central star, the dust luminosity is

$$
L_{d} / L_{\star}=A_{d} / 4 \pi a^{2}
$$

where $A_{d}$ is the cross-sectional area of the swarm of particles. For a dust albedo $w$, the luminosity in scattered light is $w L_{d} / L_{\star}$; the thermal emission is $(1-w) L_{d} / L_{\star}$.

Fig. 8 illustrates the long-term evolution of $L_{d} / L_{\star}$ for several calculations. At $250 \mathrm{AU}$, 
rings of pebbles with $f_{0}=10^{-7}$ have an initial $L_{d} / L_{\star} \approx 1-3 \times 10^{-4}$ (Fig. [8, black curve). As the system evolves, small particles merge into larger objects. The dust luminosity gradually drops, reaching $L_{d} / L_{\star} \approx 3-10 \times 10^{-7}$ after roughly $10 \mathrm{Myr}$. Near the end of the first epoch of runaway growth, the dust brightens by more than two orders of magnitude. After maintaining a peak $L_{d} / L_{\star} \approx 1-3 \times 10^{-4}$ for $50-100 \mathrm{Myr}$, the system starts to fade. During the decline, the second runaway growth phase results in a small short-lived brightening of the dust luminosity. Once runaway growth ends, the dust fades rapidly to very faint levels with $L_{d} / L_{\star} \lesssim 10^{-7}$.

In systems with more oligarchs (larger $f_{0}$ ), peak dust luminosity occurs later and later in time. For $f_{0}=10^{-4}$ (Fig. 8, violet curve), slower growth of smaller particles results in a more gradual fading of the dust luminosity. After reaching a minimum $L_{d} / L_{\star} \approx 10^{-6}$ at $300-$ $500 \mathrm{Myr}$, continued stirring by slowly growing oligarchs creates a more vigorous collisional cascade and a slowly rising $L_{d} / L_{\star}$. After 5-6 Gyr, a last gasp of runaway growth powers a steeply rising 'burst' in the dust production rate where $L_{d} / L_{\star}$ rises to $1-4 \times 10^{-3}$. As the collisional cascade continues, $L_{d} / L_{\star}$ drops.

At $750 \mathrm{AU}$, the evolution is slower and more muted. In all systems of pebbles, the dust luminosity slowly declines from an initial value of $L_{d} / L_{\star} \approx 3 \times 10^{-5}$ to $1-3 \times 10^{-7}$ at 200-300 Myr. Once runaway growth stalls, gravitational stirring by the oligarchs increases the velocities of leftover pebbles and planetesimals. Destructive collisions generate copious amounts of small particles; $L_{d} / L_{\star}$ rises by an order of magnitude and then remains roughly constant. During the second epoch of runaway growth, the dust emission rises by another order of magnitude, reaching $L_{d} / L_{\star} \approx 10^{-4}$ at $2-3 \operatorname{Gyr}\left(f_{0}=10^{-7}\right.$; Fig. 8, green curve) to $6 \operatorname{Gyr}\left(f_{0}=10^{-4}\right.$; Fig. 8 , orange curve) to $10 \operatorname{Gyr}\left(f_{0}=10^{-3}\right)$. Following runaway growth, resumption of the collisional cascade leads to a rapid drop in the dust luminosity.

\section{DISCUSSION}

Together with Bromley \& Kenyon (2014), Kenyon \& Bromley (2015), and Bromley \& Kenyon (2016), we have examined several plausible mechanisms which yield a super-Earth mass planet at 100-750 AU around a solar-type star. In scattering scenarios, multiple superEarths form at 3-20 AU (e.g., Bromley \& Kenyon 2011). As a few of these accrete gas and grow into gas giants, they scatter lower mass protoplanets into high $e$ orbits (see also Rasio \& Ford 1996; Weidenschilling \& Marzari 1996; Chatteriee et al. 2008; Ford \& Rasio 2008; Moeckel et al. 2008; Marzari et al. 2010; Nagasawa \& Ida 2011; Moeckel \& Armitage 2012, and references therein). Interactions with the gaseous disk circularize the scattered protoplanet's orbit at large $a$. The in situ models begin with a ring of solid material at $a$ $=100-750$ AU. Collisional growth produces super-Earth mass planets on time scales which depend on the semimajor axis and initial mass of the ring and the initial sizes of the solids. 
Both approaches successfully produce super-Earths with $a \approx 100-500$ AU. Scattering allows super-Earths to reach large $a$ on short time scales, $\lesssim 10 \mathrm{Myr}$. However, orbits are often eccentric, $e \gtrsim 0.1$. Although in situ growth enables more circular orbits, growth times range from $\sim 100$ Myr to $\gtrsim 10$ Gyr.

\subsection{Other Approaches to Super-Earth Formation}

Among alternatives to these scenarios, gravitational instability in a massive circumstellar disk is probably the most popular (e.g., Helled et al. 2014; Rice 2016, and references therein). Despite considerable effort to understand the formation and evolution of clumps in an unstable disk, relating specific outcomes to initial conditions remains uncertain. For super-Earth mass planets beyond 100 AU, tidally downsizing a Jupiter mass clump is es-

sential (Navakshin 2010, 2015). Achieving this goal is also uncertain (Forgan \& Rice 2013; Navakshin 2015).

Although not directly designed to address the formation of planet nine, other scattering mechanisms appear capable of placing a massive planet on a high $e$ orbit at $a \approx 250-1000$ AU. Current models for the Oort cloud rely on scattering of leftover $1 \mathrm{~km}$ and larger objects from orbits near the original locations of the gas giants (e.g., Ida et al. 2000; Morbidelli \& Levison 2004b; Brasser et al. 2006; Levison et al. 2010; Brasser et al. 2012). Dynamical interactions with nearby stars then stabilize comets within the Oort cloud. Numerical simulations of these processes often yield objects with orbits similar to Sedna, $2012 \mathrm{VP}_{113}$, and other solar system objects on high $e$ orbits with $a \gtrsim 150-200$ AU.

Other investigations consider the possibility of capturing Sedna, $2012 \mathrm{VP}_{113}$, and planet nine during a stellar flyby (e.g., Morbidelli \& Levison 2004b; Kenyon \& Bromley 2004c; Jílková et al. 2015; Li \& Adams 2016). Although numerical simulations often yield planets and dwarf planets with reasonable orbits, the trajectory and distance of closest approach for the passing star must be tuned to achieve these orbits. Some encounters also leave behind captured and indigenous objects on orbits which are inconsistent with the current inventory of trans-Neptunian objects.

All planet nine theories must consider the long-term evolution of orbits in the inner and outer solar system. Single or multiple super-Earth mass planets at $a \approx 100-300$ AU are probably inconsistent with the ephemerides of Jupiter, Saturn, and other major planets (e.g. Iorio 2012; de la Fuente Marcos \& de la Fuente Marcos 2014; Iorio 2014; Fienga et al. 2016). However, certain ranges for the true anomaly of a single super-Earth with $a \approx 500 \mathrm{AU}$ improve the Cassini residuals for Saturn (Fienga et al. 2016). If planet nine is ever detected, Cassini radio ranging data will provide a strong constraint on the allowed mass and orbital parameters.

In the outer solar system, close encounters with nearby stars can perturb the orbital 
elements of planets with $a \gtrsim 200$ AU (e.g., Morbidelli \& Levison 2004b; Kenyon \& Bromley 2004c; Brasser et al. 2012; Júlková et al. 2015; Li \& Adams 2016). Using an extensive set of numerical simulations, Li \& Adams (2016) conclude that interactions with passing stars might strip planet nine from the solar system. However, they do not address how these interactions impact the orbits of Sedna, $2012 \mathrm{VP}_{113}$, and comets within the Oort cloud. Thus, it is not clear whether typical outcomes of these simulations are consistent with observations of solar system objects.

Additional theoretical investigations are clearly needed to examine the history of the outer solar system in the context of current observations. Improvements in our understanding of disk dissipation can help us explore the evolution of gas at 100-1000 AU on time scales when collisional growth or scattering might place super-Earths in the outer disk. As disks dissipate, more detailed studies of the expansion of the inner cavity provide a way to relate the orbits of small solids and planets to the evolution of the disk. Finally, broader studies of the outcomes of stellar encounters enable a better understanding of the current architecture of the outer solar system.

\subsection{Observational Tests: Debris Disks}

Although direct imaging techniques have discovered many gas giants, they cannot detect super-Earth mass planets. However, observations of structure in debris disks place some limits on the formation of super-Earths at $a \gtrsim 100 \mathrm{AU}$ around solar-type stars (Kenyon \& Bromley 2015, and references therein). Constraints on the observed dust luminosity also provide tests of our numerical calculations.

In Kenyon \& Bromley (2015), we focused on three solar-type stars - HD 107146, HD 202628, and HD 207129 - with large rings of debris at 100-200 AU (Corder et al. 2009; Krist et al. 2010; Marshall et al. 2011; Krist et al. 2012; Ricci et al. 2015). With ages of 1-2 Gyr and relative dust luminosities $L_{d} / L_{\star} \approx 10^{-4}$, HD 202628 and HD 207129 provide interesting comparisons with our model predictions. Although the maximum dust luminosity in calculations with a single oligarch match the observations, these systems achieve peak $L_{d} / L_{\star}$ too early - $100 \mathrm{Myr}$ - and fade too rapidly. Models with 16 or more oligarchs match the observed $L_{d} / L_{\star}$ when the central star is too old, $\gtrsim 5$ Gyr. However, models with 2-8 oligarchs reach the observed $L_{d} / L_{\star}$ at 1-2 Gyr and remain bright for several Gyr. Thus, these models yield a reasonable match to observations.

Several of our calculations match the observed dust luminosity for the debris disk in the 100 Myr old star HD 107146. With $L_{d} / L_{\star} \approx 10^{-3}$ (Williams et al. 2004), this system is among the brightest debris disks around a solar-type star. Aside from our 'standard' debris disk models starting from ensembles of $1 \mathrm{~km}$ planetesimals embedded in a gaseous disk (Kenyon \& Bromley 2008, 2010), calculations of several oligarchs within a ring of pebbles at 
100-150 AU yield $L_{d} / L_{\star} \approx 10^{-3}$ at roughly $100 \mathrm{Myr}$.

Observations with ALMA will certainly improve these tests. As samples of solar-type stars with resolved debris disks beyond 100 AU grow, high spatial resolution observations should yield better comparisons with predictions of the surface density distribution. Robust estimates of the frequency and sizes of the dark lanes produced by planets can also test theoretical models.

\subsection{Observational Tests: Solar System}

Future observations will clarify the populations of planets beyond 100 AU. Current large-format optical imagers are capable of detecting planet nine and many other Sedna-like dwarf planets with an albedo of 0.05-0.3. For many of these objects, infrared detections with the James Webb Space Telescope should yield robust measurements of the albedo and radius. Within 10-15 yr, data from the Large Synoptic Survey Telescope will provide much larger samples and test our understanding of the long-term dynamics of the outer solar system.

Direct detection of planet nine clearly tests scenarios for super-Earth formation beyond 100 AU. A Super-Earth on a nearly circular orbit favors in situ formation scenarios. Eccentric orbits favor scattering models. For any orbit, the ephemerides of Jupiter, Saturn, and other gas giants place strong limits on the mass (e.g., Iorio 2014; Fienga et al. 2016).

As our understanding of the dwarf planet population at 100-1000 AU improves, comparisons of measured orbital parameters with results from long-term scattering simulations should provide tests of models with different evolutionary histories. Dwarf planets at high inclination provide a particularly stringent test, placing constraints on the initial mass in solids at large $a$ and the encounter history of the outer solar system (Jílková et al. 2015; Madigan \& McCourt 2016; Batygin \& Brown 2016; Li \& Adams 2016; Brown \& Batygin 2016).

\section{SUMMARY}

We use a suite of coagulation calculations to isolate paths for in situ production of super-Earth mass planets at 250-750 AU around solar-type stars. These paths begin with a massive ring, $M_{0} \gtrsim 15 M_{\oplus}$, composed of strong pebbles, $r_{0} \approx 1 \mathrm{~cm}$, and a few large oligarchs, $r \approx 100 \mathrm{~km}$. When these systems contain 1-10 oligarchs, two phases of runaway growth yield super-Earth mass planets in 100-200 Myr at 250 AU and 1-2 Gyr at 750 AU. Large numbers of oligarchs stir up the pebbles and initiate a collisional cascade which prevents the growth of super-Earths. For any number of oligarchs, systems of weak pebbles are also incapable of producing a super-Earth mass planet in 10 Gyr. 
The debris from swarms of pebbles producing super-Earths at 250-750 AU is directly visible. These systems have relative dust luminosities $L_{d} / L_{\star} \approx 1-30 \times 10^{-4}$ at ages of $100 \mathrm{Myr}$ to $10 \mathrm{Gyr}$. Within the rings of dust generated by planet growth, super-Earths should create gaps in the surface density distribution. Predicted widths for the gaps are 10-20 AU at $250 \mathrm{AU}$ and 30-60 AU at $750 \mathrm{AU}$.

Over the next decade, observations can test this scenario. Among exoplanetary systems, discovering super-Earths, gas giants, or debris disks far from their host stars provide vital information on the long-term evolution of protoplanets and circumstellar disks. In the solar system, orbital parameters for newly discovered dwarf planets with $a \gtrsim 100$ AU allow more rigorous tests of proposals for planet nine (e.g., Batygin \& Brown 2016; Brown \& Batygin 2016). If planet nine is real, direct detection constrains models for in situ formation and scattering (see also Bromley \& Kenyon 2014; Kenyon \& Bromley 2015; Li \& Adams 2016; Bromley \& Kenyon 2016).

We acknowledge generous allotments of computer time on the NASA 'discover' cluster. Advice and comments from M. Geller, J. Najita, and D. Wilner greatly improved our presentation. Portions of this project were supported by NASA Outer Planets Program through grant NNX11AM37G.

\section{REFERENCES}

Adachi, I., Hayashi, C., \& Nakazawa, K. 1976, Progress of Theoretical Physics, 56, 1756

Alexander, R., Pascucci, I., Andrews, S., Armitage, P., \& Cieza, L. 2014, in Protostars and Planets VI, ed. H. Beuther, R. S. Klessen, C. P. Dullemond, \& T. Henning (University of Arizona Press, Tucson, AZ), 475-496

Andrews, S. M. 2015, PASP, 127, 961

Andrews, S. M., Wilner, D. J., Espaillat, C., et al. 2011, ApJ, 732, 42

Arakawa, M., Leliwa-Kopystynski, J., \& Maeno, N. 2002, Icarus, 158, 516

Ayliffe, B. A., Laibe, G., Price, D. J., \& Bate, M. R. 2012, MNRAS, 423, 1450

Bailey, V., Meshkat, T., Reiter, M., et al. 2014, ApJ, 780, L4

Batygin, K., \& Brown, M. E. 2016, AJ, 151, 22

Benz, W., \& Asphaug, E. 1999, Icarus, 142, 5

Birnstiel, T., \& Andrews, S. M. 2014, ApJ, 780, 153 
Birnstiel, T., Dullemond, C. P., \& Brauer, F. 2010, A\&A, 513, A79+

Bottke, W. F., Nesvorný, D., Vokrouhlický, D., \& Morbidelli, A. 2010, AJ, 139, 994

Brasser, R., Duncan, M. J., \& Levison, H. F. 2006, Icarus, 184, 59

Brasser, R., Duncan, M. J., Levison, H. F., Schwamb, M. E., \& Brown, M. E. 2012, Icarus, 217,1

Brauer, F., Dullemond, C. P., \& Henning, T. 2008, A\&A, 480, 859

Bromley, B. C., \& Kenyon, S. J. 2006, AJ, 131, 2737

-. 2011, ApJ, 731, 101

-. 2014, ApJ, 796, 141

—. 2016, ApJ, submitted (available on the arXiv)

Brown, M. E., \& Batygin, K. 2016, arXiv:1603.05712

Brown, M. E., Trujillo, C., \& Rabinowitz, D. 2004, ApJ, 617, 645

Burchell, M. J., Leliwa-Kopystyński, J., \& Arakawa, M. 2005, Icarus, 179, 274

Calvet, N., D'Alessio, P., Watson, D. M., et al. 2005, ApJ, 630, L185

Canovas, H., Caceres, C., Schreiber, M. R., et al. 2016, MNRAS, 458, L29

Chatterjee, S., Ford, E. B., Matsumura, S., \& Rasio, F. A. 2008, ApJ, 686, 580

Chavanis, P. H. 2000, A\&A, 356, 1089

Chen, Y.-T., Kavelaars, J. J., Gwyn, S., et al. 2013, ApJ, 775, L8

Chiang, E., \& Youdin, A. N. 2010, Annual Review of Earth and Planetary Sciences, 38, 493

Cieza, L. A., Schreiber, M. R., Romero, G. A., et al. 2010, ApJ, 712, 925

Clarke, C. J., Gendrin, A., \& Sotomayor, M. 2001, MNRAS, 328, 485

Cloutier, R., Currie, T., Rieke, G. H., et al. 2014, ApJ, 796, 127

Corder, S., Carpenter, J. M., Sargent, A. I., et al. 2009, ApJ, 690, L65

Currie, T., Daemgen, S., Debes, J., et al. 2014a, ApJ, 780, L30

Currie, T., Lada, C. J., Plavchan, P., et al. 2009, ApJ, 698, 1

Currie, T., Muto, T., Kudo, T., et al. 2014b, ApJ, 796, L30 
Davis, D. R., Chapman, C. R., Weidenschilling, S. J., \& Greenberg, R. 1985, Icarus, 63, 30 de la Fuente Marcos, C., \& de la Fuente Marcos, R. 2014, MNRAS, 443, L59

Espaillat, C., D’Alessio, P., Hernández, J., et al. 2010, ApJ, 717, 441

Fienga, A., Laskar, J., Manche, H., \& Gastineau, M. 2016, ArXiv e-prints, arXiv:1602.06116

Ford, E. B., \& Rasio, F. A. 2008, ApJ, 686, 621

Forgan, D., \& Rice, K. 2013, MNRAS, 432, 3168

Garaud, P., Meru, F., Galvagni, M., \& Olczak, C. 2013, ApJ, 764, 146

Gibbons, P. G., Mamatsashvili, G. R., \& Rice, W. K. M. 2015, MNRAS, 453, 4232

Giblin, I., Davis, D. R., \& Ryan, E. V. 2004, Icarus, 171, 487

Gladman, B., Kavelaars, J., Petit, J.-M., et al. 2009, ApJ, 697, L91

Goldreich, P., Lithwick, Y., \& Sari, R. 2004, ARA\&A, 42, 549

Gomes, R. S., Matese, J. J., \& Lissauer, J. J. 2006, Icarus, 184, 589

Gonzalez, J.-F., Laibe, G., Maddison, S. T., Pinte, C., \& Ménard, F. 2015, Planet. Space Sci., 116,48

Gorti, U., \& Hollenbach, D. 2009, ApJ, 690, 1539

Gorti, U., Hollenbach, D., \& Dullemond, C. P. 2015, ApJ, 804, 29

Haghighipour, N., \& Boss, A. P. 2003, ApJ, 583, 996

Haisch, Jr., K. E., Lada, E. A., \& Lada, C. J. 2001, ApJ, 553, L153

Hartmann, L., Calvet, N., Gullbring, E., \& D’Alessio, P. 1998, ApJ, 495, 385

Haworth, T. J., Clarke, C. J., \& Owen, J. E. 2016, MNRAS, 457, 1905

Helled, R., Bodenheimer, P., Podolak, M., et al. 2014, in Protostars and Planets VI, ed. H. Beuther, R. S. Klessen, C. P. Dullemond, \& T. Henning (University of Arizona Press, Tucson, AZ), 643-665

Heng, K., \& Kenyon, S. J. 2010, MNRAS, 408, 1476

Holsapple, K. A. 1994, Planet. Space Sci., 42, 1067

Housen, K. R., \& Holsapple, K. A. 1999, Icarus, 142, 21

Ida, S., Larwood, J., \& Burkert, A. 2000, ApJ, 528, 351 
Iorio, L. 2012, Celestial Mechanics and Dynamical Astronomy, 112, 117

-. 2014, MNRAS, 444, L78

Jílková, L., Portegies Zwart, S., Pijloo, T., \& Hammer, M. 2015, MNRAS, 453, 3157

Johansen, A., Mac Low, M.-M., Lacerda, P., \& Bizzarro, M. 2015, Science Advances, 1, 15109

Kenyon, S. J., \& Bromley, B. C. 2001, AJ, 121, 538

-. 2002, ApJ, 577, L35

-. 2004a, AJ, 127, 513

-. 2004b, ApJ, 602, L133

-. 2004c, Nature, 432, 598

—. 2004d, AJ, 128, 1916

-. 2006, AJ, 131, 1837

-. 2008, ApJS, 179, 451

-. 2010, ApJS, 188, 242

-. 2012, AJ, 143, 63

-. 2015, ApJ, 806, 42

-. 2016, ApJ, 817, 51

Kenyon, S. J., Gómez, M., \& Whitney, B. A. 2008, in Handbook of Star Forming Regions, Volume I, ed. Reipurth, B., 405-458

Kenyon, S. J., \& Hartmann, L. 1995, ApJS, 101, 117

Kenyon, S. J., \& Luu, J. X. 1998, AJ, 115, 2136

-. 1999, AJ, 118, 1101

Kim, K. H., Watson, D. M., Manoj, P., et al. 2013, ApJ, 769, 149

Klahr, H. H., \& Henning, T. 1997, Icarus, 128, 213

Kobayashi, H., \& Tanaka, H. 2010, Icarus, 206, 735

Kobayashi, H., Tanaka, H., Krivov, A. V., \& Inaba, S. 2010, Icarus, 209, 836 
Krist, J. E., Stapelfeldt, K. R., Bryden, G., \& Plavchan, P. 2012, AJ, 144, 45

Krist, J. E., Stapelfeldt, K. R., Bryden, G., et al. 2010, AJ, 140, 1051

Lafrenière, D., Jayawardhana, R., \& van Kerkwijk, M. H. 2010, ApJ, 719, 497

Lagrange, A.-M., Bonnefoy, M., Chauvin, G., et al. 2010, Science, 329, 57

Leinhardt, Z. M., \& Stewart, S. T. 2009, Icarus, 199, 542

—. 2012, ApJ, 745, 79

Leinhardt, Z. M., Stewart, S. T., \& Schultz, P. H. 2008, in The Solar System Beyond Neptune, ed. Barucci, M. A., Boehnhardt, H., Cruikshank, D. P., \& Morbidelli, A. (University of Arizona Press, Tucson, AZ), 195-211

Levison, H. F., Duncan, M. J., Brasser, R., \& Kaufmann, D. E. 2010, Science, 329, 187

Li, G., \& Adams, F. C. 2016, ArXiv e-prints, arXiv:1602.08496

Lissauer, J. J. 1987, Icarus, 69, 249

Love, S. G., \& Ahrens, T. J. 1996, Icarus, 124, 141

Lykawka, P. S., \& Mukai, T. 2008, AJ, 135, 1161

Madigan, A.-M., \& McCourt, M. 2016, MNRAS, 457, L89

Mamajek, E. E. 2009, in American Institute of Physics Conference Series, Vol. 1158, American Institute of Physics Conference Series, ed. T. Usuda, M. Tamura, \& M. Ishii, $3-10$

Marois, C., Macintosh, B., Barman, T., et al. 2008, Science, 322, 1348

Marshall, J. P., Löhne, T., Montesinos, B., et al. 2011, A\&A, 529, A117

Marzari, F., Baruteau, C., \& Scholl, H. 2010, A\&A, 514, L4

Matese, J. J., Whitman, P. G., \& Whitmire, D. P. 1999, Icarus, 141, 354

Michikoshi, S., Inutsuka, S.-i., Kokubo, E., \& Furuya, I. 2007, ApJ, 657, 521

Michikoshi, S., Kokubo, E., \& Inutsuka, S.-i. 2009, ApJ, 703, 1363

-. 2010, ApJ, 719, 1021

Moeckel, N., \& Armitage, P. J. 2012, MNRAS, 419, 366

Moeckel, N., Raymond, S. N., \& Armitage, P. J. 2008, ApJ, 688, 1361 
Morbidelli, A., \& Levison, H. F. 2004a, AJ, 128, 2564

-. 2004b, AJ, 128, 2564

Nagasawa, M., \& Ida, S. 2011, ApJ, 742, 72

Najita, J. R., Andrews, S. M., \& Muzerolle, J. 2015, MNRAS, 450, 3559

Najita, J. R., \& Kenyon, S. J. 2014, MNRAS, 445, 3315

Najita, J. R., Strom, S. E., \& Muzerolle, J. 2007, MNRAS, 378, 369

Nayakshin, S. 2010, MNRAS, 408, L36

-. 2015, MNRAS, 454, 64

O’Brien, D. P., \& Greenberg, R. 2003, Icarus, 164, 334

Ohtsuki, K. 1999, Icarus, 137, 152

Ohtsuki, K., Stewart, G. R., \& Ida, S. 2002, Icarus, 155, 436

Owen, J. E., Clarke, C. J., \& Ercolano, B. 2012, MNRAS, 422, 1880

Owen, J. E., Hudoba de Badyn, M., Clarke, C. J., \& Robins, L. 2013, MNRAS, 436, 1430

Rabinowitz, D., Schwamb, M. E., Hadjiyska, E., Tourtellotte, S., \& Rojo, P. 2013, AJ, 146, 17

Raettig, N., Klahr, H., \& Lyra, W. 2015, ApJ, 804, 35

Rafikov, R. R. 2004, AJ, 128, 1348

Rasio, F. A., \& Ford, E. B. 1996, Science, 274, 954

Ricci, L., Carpenter, J. M., Fu, B., et al. 2015, ApJ, 798, 124

Rice, K. 2016, ArXiv e-prints, arXiv:1602.08390

Ryan, E. V., Davis, D. R., \& Giblin, I. 1999, Icarus, 142, 56

Sheppard, S. S. 2010, AJ, 139, 1394

Simon, J. B., Armitage, P. J., Li, R., \& Youdin, A. N. 2015, ArXiv e-prints, arXiv:1512.00009

Stern, S. A., \& Colwell, J. E. 1997, AJ, 114, 841

Tanaka, H., Inaba, S., \& Nakazawa, K. 1996, Icarus, 123, 450

Tobin, J. J., Looney, L. W., Wilner, D. J., et al. 2015, ApJ, 805, 125 
Trujillo, C. A., \& Sheppard, S. S. 2014, Nature, 507, 471

Weidenschilling, S. J. 1977, MNRAS, 180, 57

-. 1989, Icarus, 80, 179

—. 2010, ApJ, 722, 1716

Weidenschilling, S. J., \& Marzari, F. 1996, Nature, 384, 619

Wetherill, G. W., \& Stewart, G. R. 1993, Icarus, 106, 190

Williams, D. R., \& Wetherill, G. W. 1994, Icarus, 107, 117

Williams, J. P., \& Cieza, L. A. 2011, ARA\&A, 49, 67

Williams, J. P., Najita, J., Liu, M. C., et al. 2004, ApJ, 604, 414

Windmark, F., Birnstiel, T., Ormel, C. W., \& Dullemond, C. P. 2012, A\&A, 544, L16

Youdin, A. N., \& Chiang, E. I. 2004, ApJ, 601, 1109

Youdin, A. N., \& Kenyon, S. J. 2013, From Disks to Planets, ed. T. D. Oswalt, L. M. French, \& P. Kalas (Dordrecht: Springer Science \& Business Media), 1

Youdin, A. N., \& Shu, F. H. 2002, ApJ, 580, 494

Zhu, Z., Stone, J. M., Rafikov, R. R., \& Bai, X.-N. 2014, ApJ, 785, 122 
Table 1. List of Variables

\begin{tabular}{|c|c|}
\hline Variable & Definition \\
\hline$a$ & semimajor axis, radial coordinate \\
\hline$a_{\text {in }}$ & semimajor axis of the inner edge of the disk \\
\hline$a_{\max }$ & semimajor axis of the maximum pressure in the disk \\
\hline$a_{\text {out }}$ & semimajor axis of the outer edge of the disk \\
\hline$A_{d}$ & cross-sectional area of particles \\
\hline$b_{d}$ & exponent in relation for debris from collisions \\
\hline$b_{L}$ & exponent in relation for mass of largest particle in debris \\
\hline$e$ & eccentricity \\
\hline$e_{\text {rel }}$ & eccentricity relative to the Hill radius of the largest object \\
\hline$f_{g}$ & gravitational focusing factor \\
\hline$f_{0}$ & fraction of initial mass in oligarchs \\
\hline$h$ & horizontal velocity \\
\hline$i$ & inclination \\
\hline$L_{d}$ & reprocessed stellar luminosity of solid particles \\
\hline$L_{\star}$ & stellar luminosity \\
\hline$m, m_{k}$ & particle mass \\
\hline $\bar{n}$ & average mass of particle in a mass bin \\
\hline$m_{\max , d}$ & mass of largest particle in debris \\
\hline$m_{L, 0}$ & coefficient in relation for mass of largest particle in debris \\
\hline$m_{\text {esc }}$ & mass of debris ejected in a collision \\
\hline$m_{\max }$ & mass of largest particle in the grid \\
\hline$m_{\min }$ & mass of smallest particle in the grid \\
\hline$M_{0}$ & total initial mass in particles \\
\hline$M_{\star}$ & stellar mass \\
\hline$N, N_{k}$ & particle number \\
\hline$N_{\max }$ & number of largest particles \\
\hline$P$ & gas pressure in the disk \\
\hline$P_{\max }$ & maximum gas pressure in the disk \\
\hline$q_{p}$ & perihelion distance \\
\hline$Q_{b}, Q_{g}$ & coefficients in $Q_{D}^{\star}$ relation \\
\hline$Q_{c}$ & center of mass collision energy \\
\hline$Q_{D}^{\star}$ & collision energy required to eject $50 \%$ of the mass \\
\hline$r, r_{k}$ & particle radius \\
\hline $\bar{r}$ & average radius of particle in a mass bin \\
\hline$r_{\max }$ & radius of largest particle \\
\hline$r_{\min }$ & radius of smallest particle \\
\hline
\end{tabular}


Table 1-Continued

\begin{tabular}{ll}
\hline \hline Variable & \multicolumn{1}{c}{ Definition } \\
\hline$R_{\star}$ & radius of central star \\
$t$ & time \\
$T$ & temperature \\
$v$ & vertical velocity \\
$v_{c}$ & relative collision velocity \\
$v_{K}$ & orbital velocity \\
$V$ & volume \\
$\beta_{b}, \beta_{g}$ & exponents in $Q_{D}^{\star}$ relation \\
$\delta$ & mass spacing factor \\
$\delta a$ & width of annulus \\
$\rho_{p}$ & particle mass density \\
$\sigma$ & geometric cross section \\
$\Sigma$ & surface density \\
$\omega$ & argument of perihelion \\
$\Omega$ & angular velocity \\
$\varpi$ & longitude of perihelion \\
\hline
\end{tabular}

Note. - Variables with a subscript ' 0 ' refer to initial conditions; e.g., $e_{0}$ is the initial eccentricity 


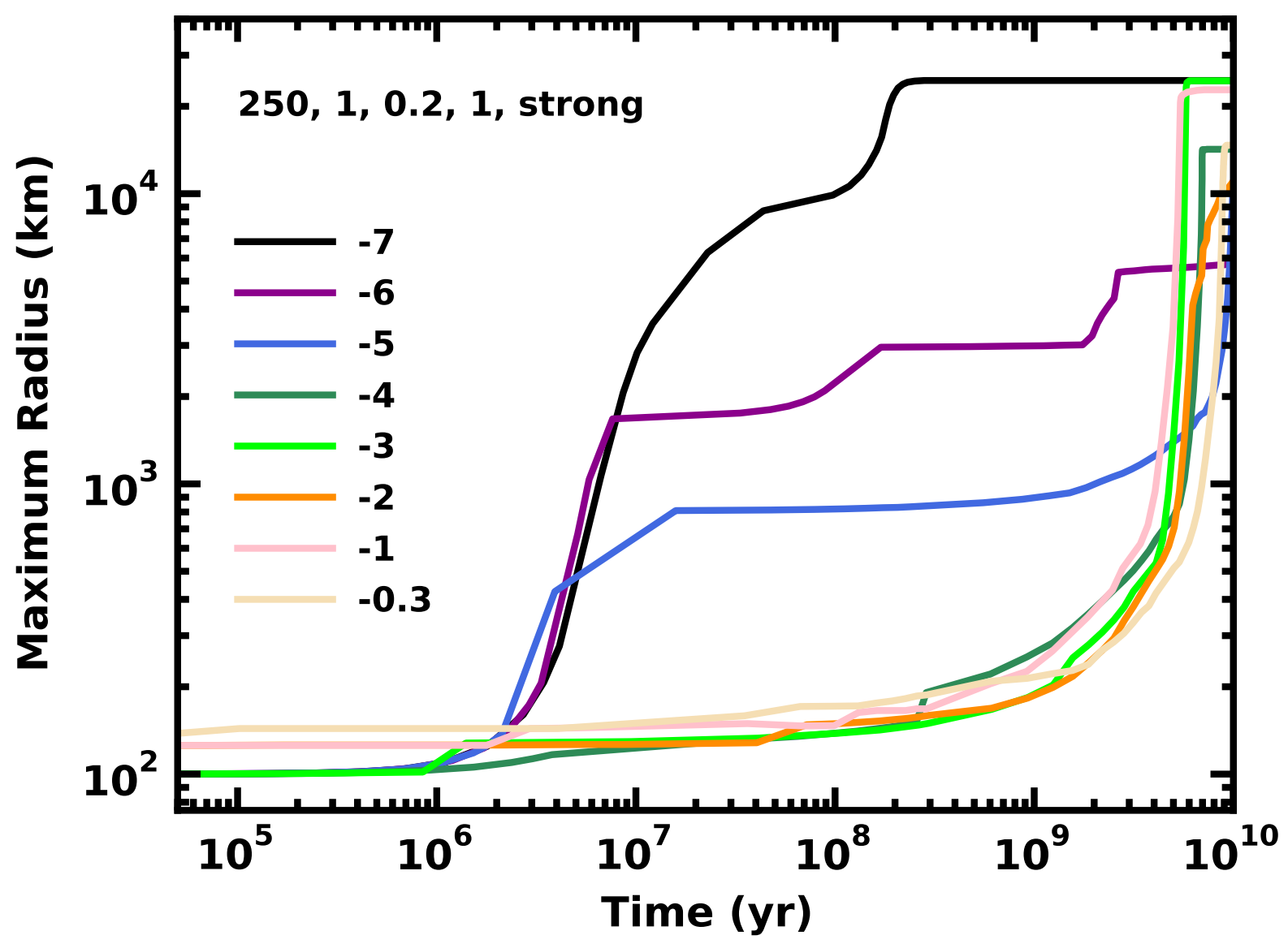

Fig. 1. - Growth of the largest object at $250 \mathrm{AU}$ as a function of $f_{0}$, the initial mass fraction of solid material in $100 \mathrm{~km}$ objects, for calculations with $b_{d}=1, m_{L, 0}=0.2, b_{L}=1$, and the strong fragmentation parameters. The legend indicates log $f_{0}$ for each calculation. When $f_{0} \lesssim 10^{-5}$, large objects grow rapidly; sometimes, these objects reach super-Earth masses with $r_{\max } \gtrsim 10^{4} \mathrm{~km}$ on short time scales. When $f_{0} \gtrsim 10^{-4}$, the largest objects grow slowly to super-Earth masses on time scales of 5-10 Gyr. 


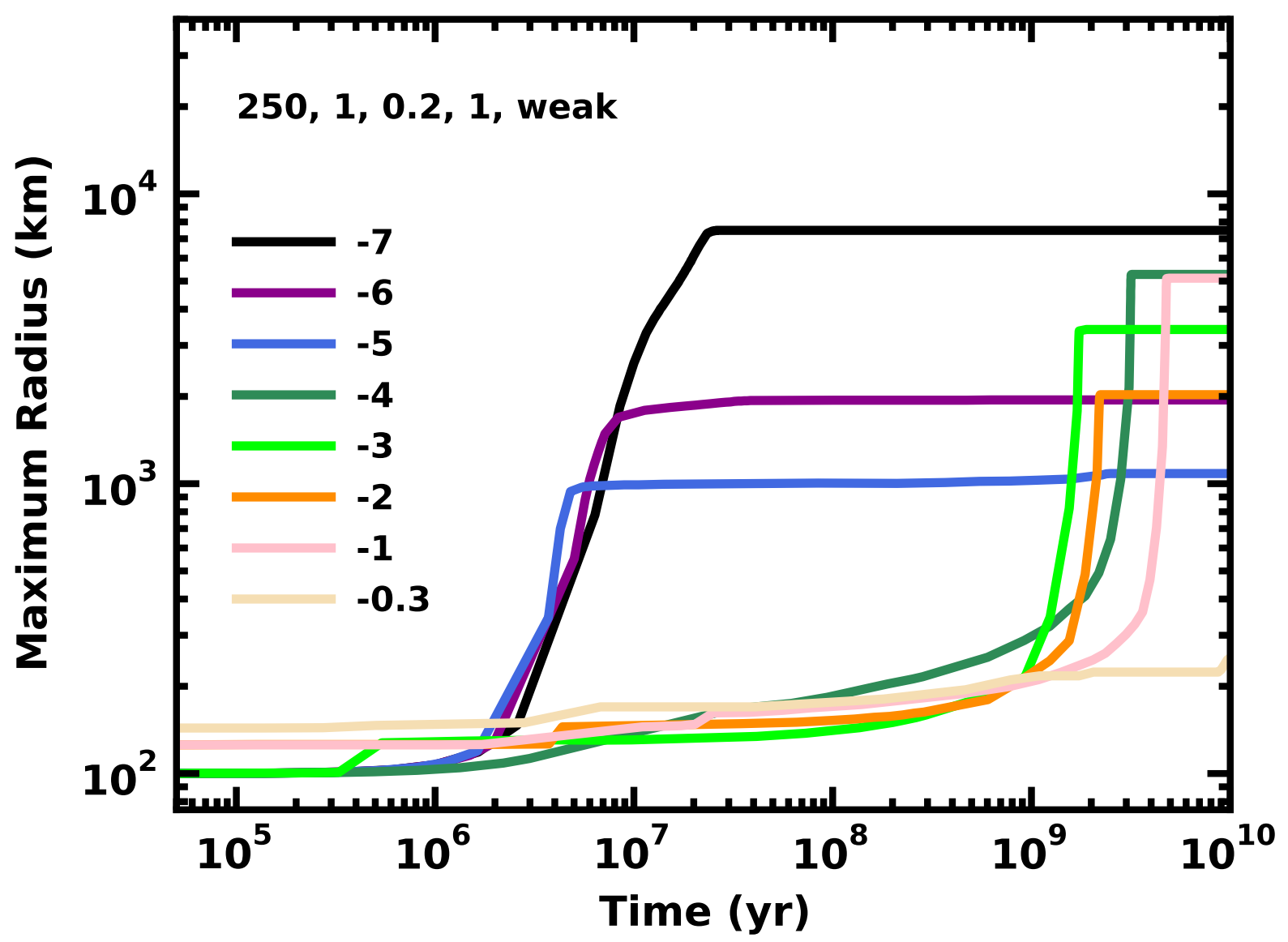

Fig. 2.- As in Fig. 1 for calculations with the weak fragmentation parameters. When the initial $f_{0}$ is small (large), growth yields larger (smaller) planets. However, these systems rarely produce super-Earth mass planets. 


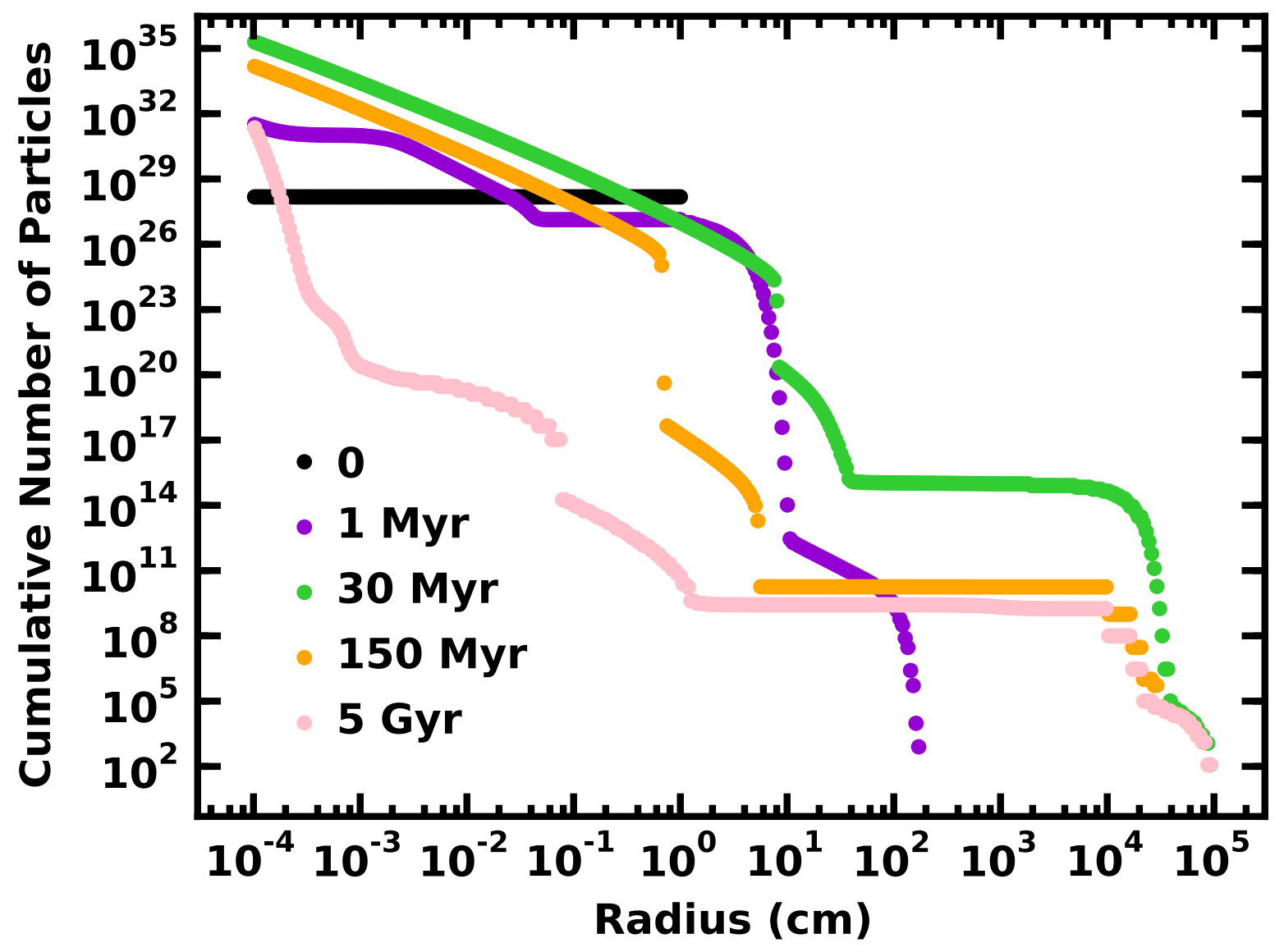

Fig. 3.- Time evolution of the cumulative size distribution for small particles with $r \lesssim$ $1 \mathrm{~km}$ at $250 \mathrm{AU}$ in a simulation with $f_{0}=10^{-7}, b_{d}=1, m_{L, 0}=0.2, b_{L}=1$, and strong planetesimals. 


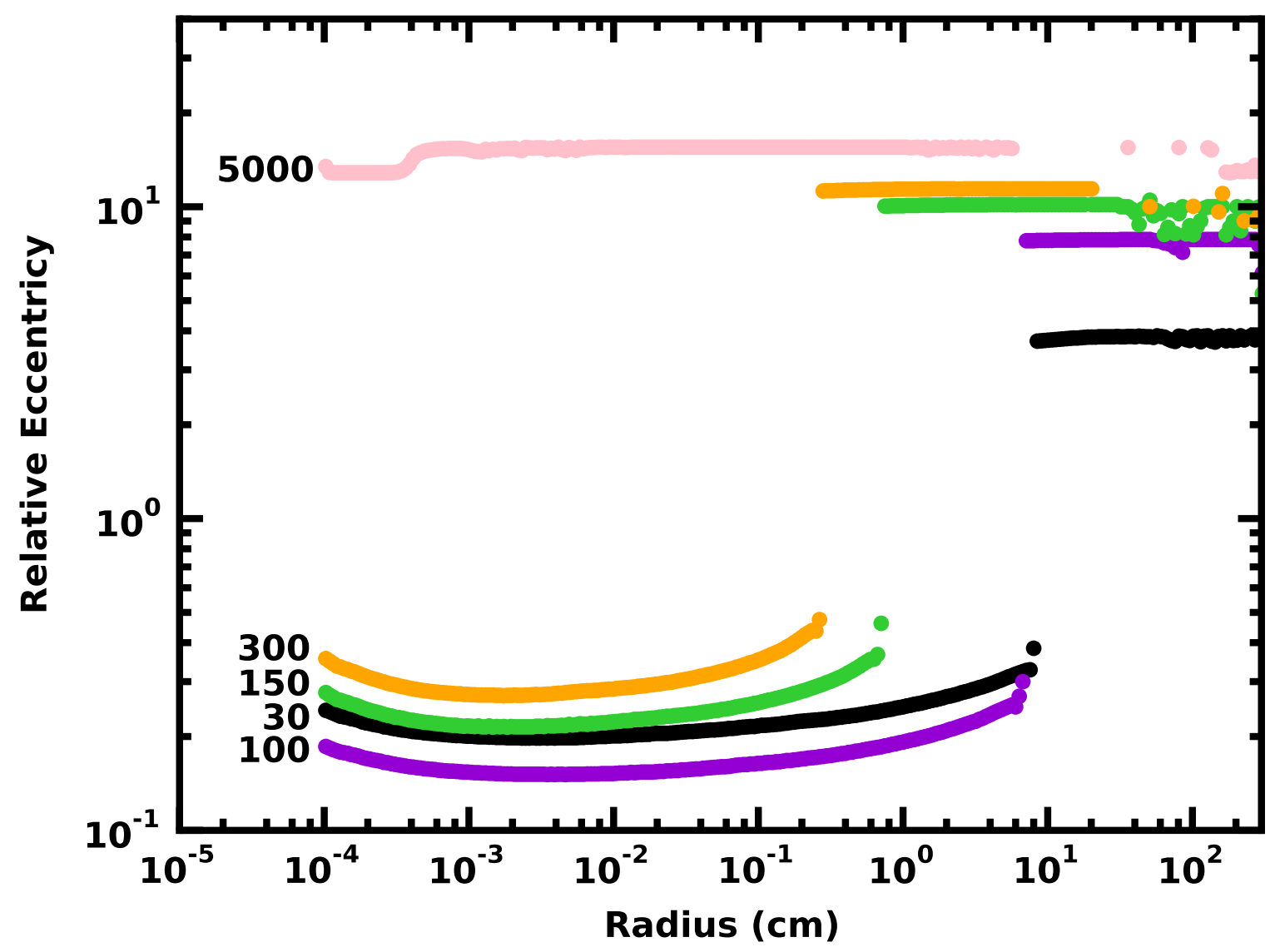

Fig. 4.- As in Fig. 3 for the time evolution of the relative eccentricity distribution $\left(e_{r e l}=\right.$ $e a / R_{H}$, where $R_{H}$ is the Hill radius of the largest oligarch) for small particles with $r \lesssim 1 \mathrm{~m}$ at $250 \mathrm{AU}$. Numbers to the left of each track indicate the evolution time in Myr. 


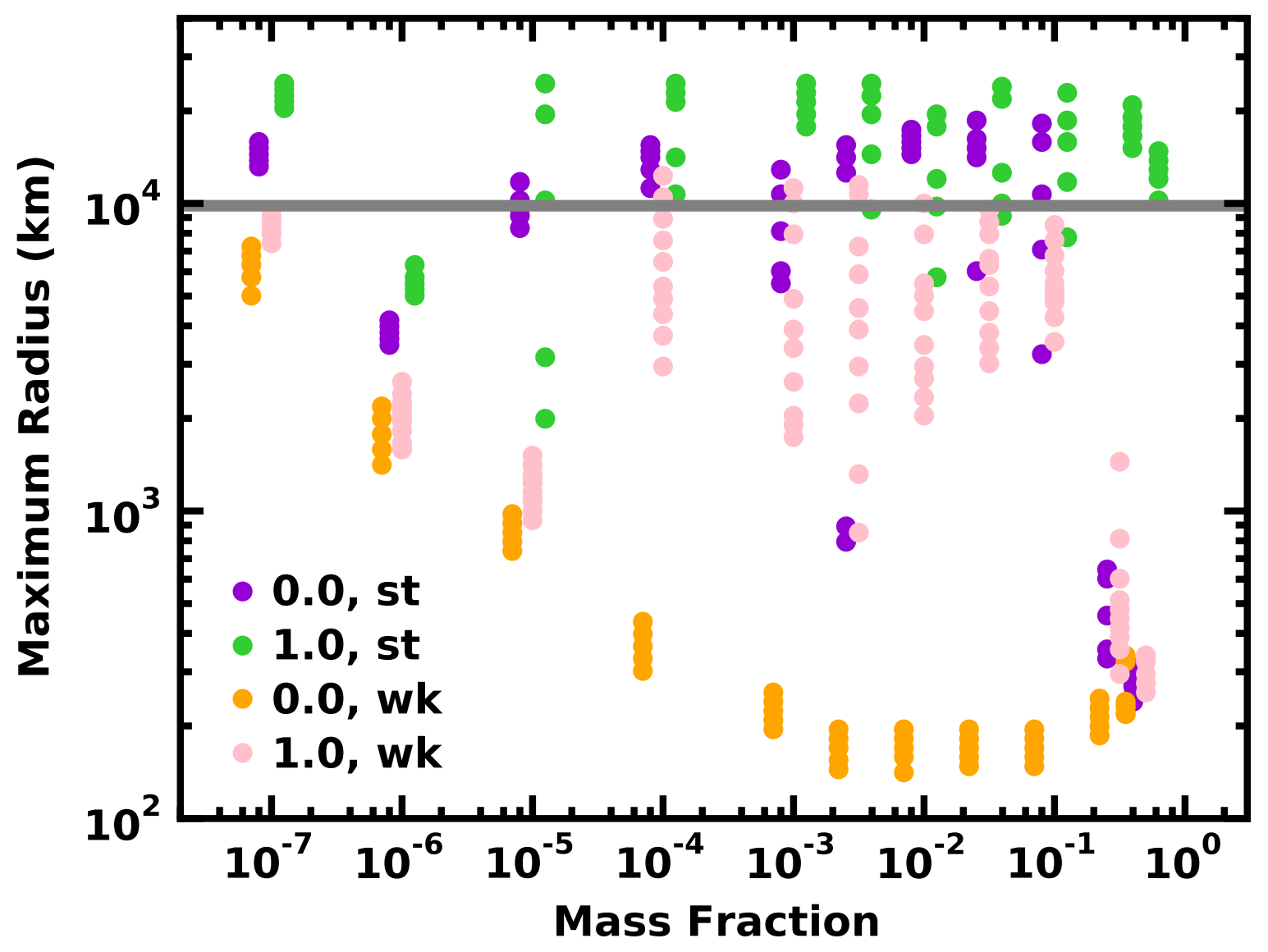

Fig. 5.- Maximum radius $r_{\max }$ as a function of the mass fraction $f_{0}, b_{L}$, and the strength of small planetesimals for calculations at $250 \mathrm{AU}$. The legend indicates $b_{L}$ and the planetesimal strength ('st' for strong; 'wk' for weak). Super-Earth formation generally requires strong planetesimals. 


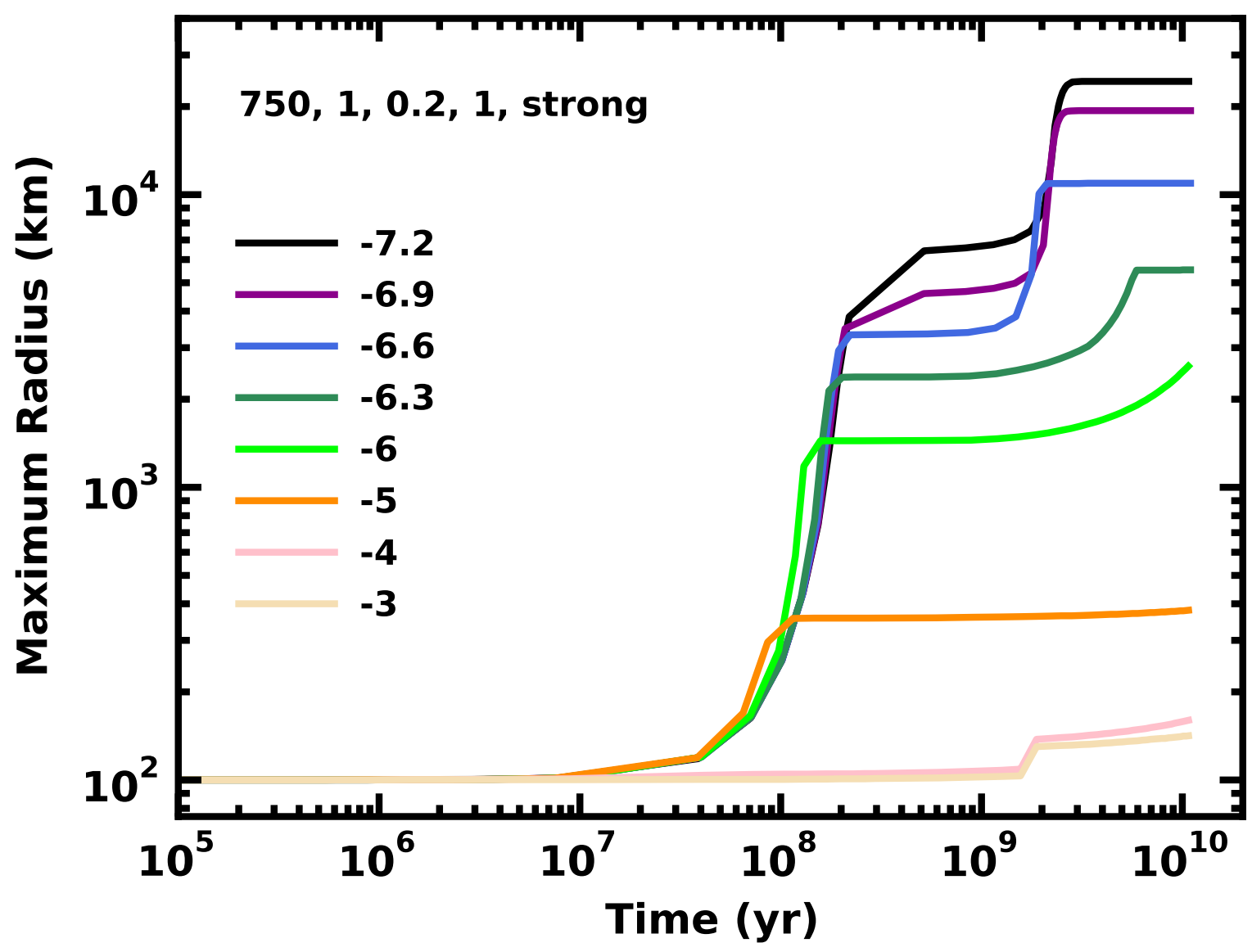

Fig. 6. - Growth of the largest object at $750 \mathrm{AU}$ as a function of $f_{0}$, the initial mass fraction of solid material in $100 \mathrm{~km}$ objects, for calculations with $b_{d}=1, m_{L, 0}=0.2, b_{L}=1$, and the strong fragmentation parameters. The legend indicates $\log f_{0}$. Simulations with $f_{0} \lesssim 10^{-5}$ produce $300-4000 \mathrm{~km}$ objects in 100-200 Myr. After a 1-5 Gyr period where the largest objects grow very slowly, simulations with $f_{0} \lesssim 5 \times 10^{-7}$ undergo a second phase of runaway growth. The largest objects may then reach super-Earth sizes with $r_{\max } \gtrsim 10^{4} \mathrm{~km}$. 


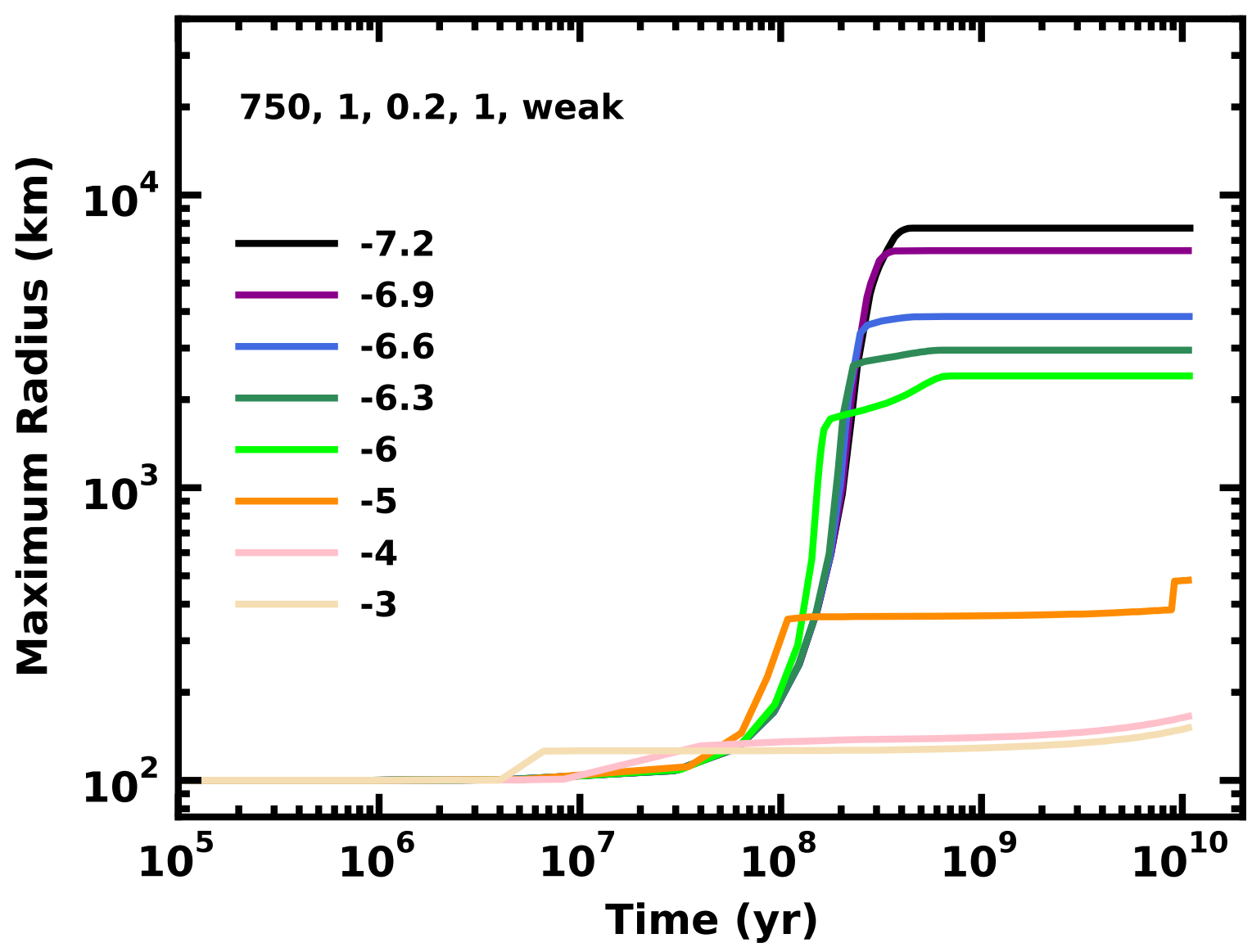

Fig. 7.- As in Fig. 6 for calculations with weak planetesimals. Although $r_{\max }$ correlates inversely with the initial mass in oligarchs, the largest objects never reach super-Earth masses. 


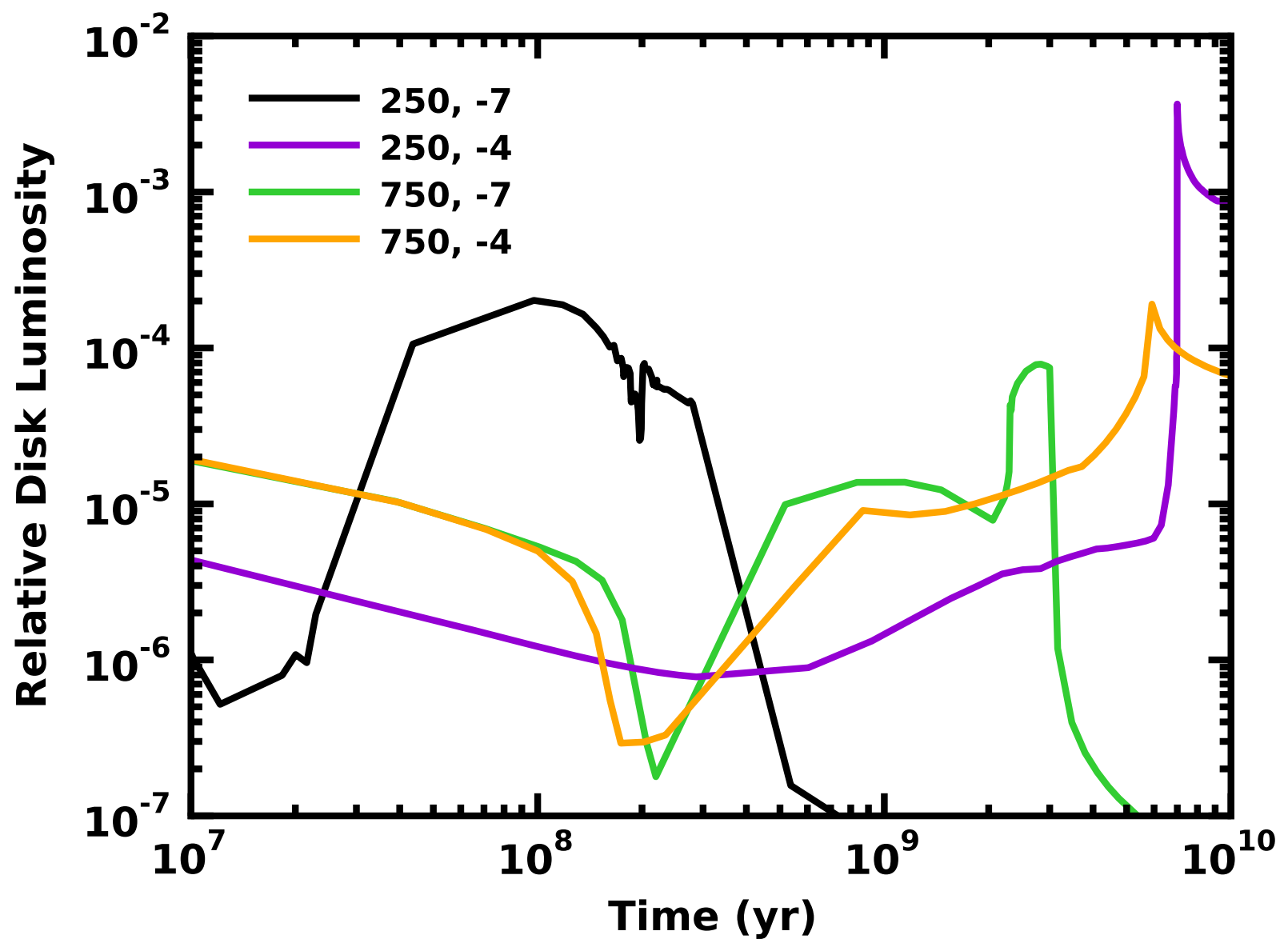

Fig. 8.- Evolution of the relative dust luminosity $L_{d} / L_{\star}$ for calculations at 250-750 AU. The legend indicates $a$ and $\log f_{0}$ for each model. Typical maximum dust luminosities range from $L_{d} / L_{\star} \approx 10^{-5}-10^{-3}$ at $100 \mathrm{Myr}$ to $L_{d} / L_{\star} \approx 10^{-9}-10^{-3}$ at $10 \mathrm{Gyr}$. 